\title{
MÉDIAS DE CURVATURAS EM VARIEDADES FOLHEADAS
}

FABIANO GUSTAVO BRAGA BRITO

TESE APRESENTADA

AO

INSTITUTO DE MATEMATIICA E ESTATISTICA

DA

UNIVERSIDADE DE SÄO PAULO

PARA OBTENÇĀO DO GRAU DE DOUTOR

EM

MATEMAT ICA

ORIENTADOR: Prof. Dr. Carlos Edgard Harle

- SĀo PAUlo, SETEMbro de 1981 - 
observa-se, no estudo geometrico das variedades bolheadas dois pontos de vista principais. Podemos dizer que o primeiro è quantitativo e relaciona-se com o problema de saber, por exemplo, quantas classes de isotopia de folheaçöes existem num dado espaço com características topológicas determinadas. A ferramenta principal para tal anälise são as classes caracterỉsticas de folheaçöes.

o segundo ponto de vista è qualitativo e trata, enthe outras coisas de determinar propriedades geometricas das folheaçöes numa dada variedade riemanniana.

os resultados apresentados neste trabalho são quali tativos.

No primeiro capêtulo apresentamos uma demonstraçäo elementar de um teorema de D. Asimov que foi generalizado por R. Langevin, H.Rosenberg e pelo autor desta tese. Trata-se de saber como variam as medias das curvaturas de uma folheaçäo de codimensão 1 sobre uma variedade compacta, sem bordo e de curvatura seccional constante.

D. Asimov provou que se $w^{n+1} \bar{e}$ uma tal variedade fo lheada, então

$$
\frac{1}{\operatorname{vol}(w)} \int_{\omega} K=\lambda(c, n),
$$

- onde $\lambda(c, n) \bar{e}$ um nümero que depende somente da curvatura c (constante) e da dimensção $n$ das folhas. K representa a curvatura de Lipschitz-Killing lintegrando do teorema de Gauss-Bonnet-chern) das folhas, calculados em cada ponto de $w$. 
Existem outras funções de curvatura definidas para as folhas que s $\vec{o}$ dependem da metrica nelas induzidas Ipor exemplo a curvatura escalar). Provaremos que as médias de tais curvaturas tambèm não dependem da jolheaçäo.

No segundo capêtulo são consideradas jolheações de codimensão 1 em espaços de curvatura näo necessariamente cons tante.

Os resultados $a \vec{i}$ obtidos generalizam os do primeiro capêtulo em duas direções principais:

19.) Fornece limitações para a média da curvatura escalar em funçäo de limitações impostas à variação do tensor de curvatura.

Precisamente, se $c_{i j}(x) \bar{e}$ a curvatura seccional de W no ponto $x$ e na direção do plano gerado pelos vetores $e_{i}, e_{j}$ e se existem $\alpha, \beta \in \mathbb{R}$ tais que $\alpha \leq c_{i j}(x) \leq \beta, \forall e_{i}, e_{j}, \forall{ }_{x}, e \underline{n}$ tão

$$
\lambda(\alpha, n) \leq \frac{1}{\operatorname{vol}(\omega)} \int_{\omega} r_{2} \leq \lambda(\beta, n),
$$

onde $\gamma_{2} \bar{e}$ a curvatura escalar das folhas $e \lambda(\alpha, n), \lambda(\beta, n)$ s $\bar{o}$ dependem de $\alpha, \beta$ e da dimensão $n$ das folhas.

29) Generaliza completamente o teorema principal do 19 capitulo para o caso da curvatura escalar das folhas em espaços de Einstein, ou seja,

- se a variedade ambiente $\omega^{n+1}$ e Einstein /curvatura de Ricci constantel então a média das curvaturas escalares das folhas goza da mesma propriedade de invariância que os espaços de curvatura constante.

Alēm disso, obtivemos uma obstruçäo geométrica à e xistência de folheaçöes totalmente geodösicas de codimensäo 1 . 
Isto acontece quando o espaço ambiente $\omega^{n+1}$ possui uma das cur vaturas seccionais generalizadas (curvatura p-seccional) não nula em todos os pontos e em todas as direções.

No capitulo 3 existem generalizaçöes parciais dos resultados precedentes para o caso de folheações ortogonais bem camo um teorema de obstrução à existência de folheações mênimas (folheações cujas folhas são subvariedades minimas de $W$ ).

Provaremos que a soma das mēdias das curvaturas $\bar{e}$ invariante com relação às folheaçöes nos seguintes casos:

a) Quando as bolheações têm codimensão 1 , são duas a duas perpendiculares e são tantas quanto a dimensão de $W$.

b) No caso de folheações ortogonais de dimensöes com plementares em espaços de curvatura constante.

Para as bolheações minimas apresentamos uma obstrução topolōgica à integrabilidade da distribuição normal quando o espaço ambiente tem curvatura positiva e a codimensão da folheação $\bar{e}$ dois.

o método utilizado na demonstração dos resultados dos capítulos 2 e 3 e o método do triedro mōvel de Elie cartan.

Estaremos sempre supondo que as variedades, métricas e bolheações envolvidas são de classe $c^{\infty}$ e sem singularida des.

As variedades onde estäo definidas as folheações se rão sempre variedades riemannianas compactas, conexas, orientā veis e de bordo vazio mesmo que tais condiçöes não apareçam ex plicitas nos enunciados. 
- A Rémi Langevin, pelas valiosas idéias e contribuições matemāticas dele recebidas na elaboraçäo deste trabatho. Sua cooperação e encorajamento foram cruciais para o bom termo deste manuscrito.

- Agradecimentos mais do que merecidos ao meu orientador Carlos Edgard Harle. As folheaçöes mënimas näo seriam aqui mencionadas não fosse a atividade denodada deste probessor que tanto se esforça por ensinar como por aprender.

- A todos os professores do IMEUSP e em particular à Proba. Elza Gomide, que me guiou nos primeiros passos do aprendizado e da pesquisa matemätica.

- A H.Rosenberg por ter dirigido o trabalho que deu origem a esta tese. 
Sôbre um Teorema devido a D.Asimov. Uma versão Geọ métrica

\section{$\S 1-$ Apresentação}

Seja $\mathrm{W}^{\mathrm{n}+1}$ uma variedade riemanniana compacta sem bordo de dimensão $n+1$ e curvatura seccional constante igual a c.

Seja $F$ uma folheação de codimensão 1 transversalmente orientāvel sobre $W^{n+1}$ e $K: W^{n+1} \rightarrow \mathbb{R}$ a função que associa a cada ponto $x \in W$ a curvatura de Lipschitz-Killing no ponto $x$ da folha que passa por $x$ munida da mêtrica induzida pela inclusão em $W^{n+1}$.

D. Asimov demonstrou em [1] que $\int_{W} K$ não depende da folheação. Em particular,

$$
\frac{1}{\operatorname{vol}(W)} \int_{W} K=\left\{\begin{array}{l}
0 \text { se } n \text { é impar } \\
\frac{2^{n} c^{\frac{n}{2}}}{\left(\frac{n}{2}\right)} \text { se } n \text { è par }
\end{array}\right.
$$

Por outro 1ado, R.Langevin, H.Rosenberg e o autor deste trabalho demonstraram em [2] que

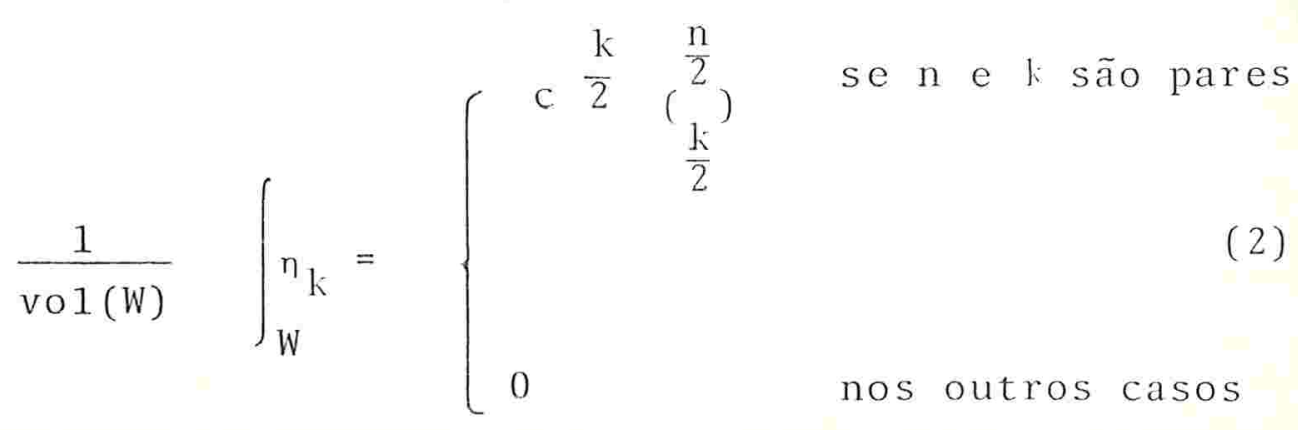

onde $\eta_{k}$ designa a k-ësima função simētrica de curvatura extrín 
seca.

As funções $n_{k}$ são definidas por

$$
\operatorname{det}(I+t A x)=\sum_{i=1}^{n} n_{i}(x) t^{i}
$$

onde:

I $\bar{e}$ o endomorfismo identidade $T_{x}{ }^{M} x \rightarrow T_{x}{ }^{M} x$

$$
A_{x}: T_{x} M_{x} \rightarrow T_{x} M_{x} \text { é dada por }
$$

$$
\begin{aligned}
& A_{X}(Y)=\nabla_{Y}(N), \\
& , M_{X} \vec{e} \text { a folha que passa por } x, \\
& , \quad \nabla \vec{e} \text { a conexão riemanniana de } W .
\end{aligned}
$$

Suponhamos agora que $n+1$ é impar $(n+1$ è a dimensão de W) :

A partir de (2), prova-se em [2] que o teorema de Asimov continua verdadeiro se substituirmos K (curvatura de Lipschitz-Killing das folhas) por $r_{2 k}$ (curvatura $2 \mathrm{k}$-escalar das folhas). Precisamente, teremos:

$$
\frac{1}{\operatorname{vol}(W)} \int_{W} \gamma_{2 k}=\sum_{x=0} c k \frac{\left(c^{k}\right)\left(\begin{array}{c}
\frac{n}{2} \\
r
\end{array}\right.}{n}
$$

A curvatura $2 k$-escalar de uma variedade riemanniana è a generalização natural da curvatura escalar quando consideramos curvaturas seccionais generalizadas (curvatura $2 k$. -seccional) e ela mede em cada ponto da variedade a média das 
curvaturas $2 \mathrm{k}$-seccionais quando fazemos variar o $2 k-1$ ano cor respondente na grassmaniana dos $2 \mathrm{k}-p 1$ planos associada aos espaço tangente (Ver [12]).

Pode-se verificar facilmente que $r_{2 k}$ è uma combina ção linear dos $n_{i}$ onde os coeficientes dependem somente das dimensões envolvidas ( $k$ e $n$ ) e da curvatura seccional (cons tante) do espaço ambiente, c. Daí o fato de a förmula (3) decorrer da fórmula (2).

Apresentamos neste capítulo uma demonstração "geométrica" dos resultados acima. Tal demonstração nos foi comunicada por R.Langevin e serviră de motivação para os longos câlculos que serão feitos no capítulo II onde será estudado o caso onde a curvatura de $W$ não é constante.

\section{$\S 2$ - Uma Demonstração Geomëtrica do Teorema de As $\underline{i}$ mov .}

Lembramos que uma variedade riemanniana compacta conexa e sem bordo de curvatura seccional constante c e dimen são n è um dos seguintes espaços:

a) Quociente do toro $\mathrm{T}^{\mathrm{n}}$, se $\mathrm{c}=0$

b) Quociente da esfera $S_{C}^{n}$ de curvatura $c>0$ pela ação de um subgrupo fechado e discreto do grupo de isometrias de $\mathrm{S}_{\mathrm{C}}^{\mathrm{n}}$, se $\mathrm{c}>\mathrm{O}$

c) Idem para o espaço hiperbölico $\mathrm{H}_{\mathrm{C}}^{\mathrm{n}}$ de curvatura c, se $c<0$.

Tais resultados podem ser vistos, por exemplo, em [14].

Em vista disso, a demonstração se dividirá em 3 par tes. 


\section{$2.1-0$ caso $c=0$}

Seja $W^{n+1}$ variedade riemanniana compacta conexa sem bordo de dimensão $n+1$ e curvatura seccional constante igual a zero. Seja $F$ uma folheação de codimensão 1 sobre W. Sejam $\eta_{\mathrm{k}}: \mathrm{W} \rightarrow \mathbb{R}, \mathrm{k}=0,1 \ldots, \mathrm{n}$, a função que associa a cada ponto $\mathrm{x} \in \mathbb{W}$ a k-êsima função simétrica de curvatura extrínseca no ponto $x$ da folha que passa por $x$. E fäcil ver que $\sigma_{k}$ só depende da mê trica induzida na folha pela métrica de W. Isto se dá porque - espaço ambiente $W^{n+1}$ curva constantemente. (Ver [5], |6」).

Como $W$ tem curvatura seccional nula, podemos cons $\underline{i}$ derar a aplicação quociente.

$$
\Gamma: \mathrm{T}^{\mathrm{n}+1} \rightarrow \mathrm{W}^{\mathrm{n}+1}=\frac{\mathrm{T}^{\mathrm{n}+1}}{\mathrm{~F}}
$$

que nos dá uma isometria local onde l: representa um subgrupo finito do grupo de isometrias de $\mathrm{T}^{\mathrm{n}+1}$.

A folheação $F$ se "levanta" naturalmente para $\mathrm{T}^{\mathrm{n}+1}$.

Seja $F^{*}=\Gamma^{*}(F)$ o levantamento de $F$ em $T^{n+1}$.

Como as funções $n_{k}$ sö dependem da mëtrica das fothas e $\Gamma$ e uma isometria local, temos:

$n_{\mathrm{k}}^{*}=n_{k} \circ \Gamma$ onde $n_{k}^{*}$ è a k-ësima função simêtrica de curvatura extrínseca de $F^{*}$.

Assim,

$$
\int_{T}^{n+1} n_{k}^{*}=m \int_{W}^{n} k
$$

onde $m$ è nümero de elementos de F.

$$
\text { Como } \operatorname{vol}\left(\mathrm{T}^{\mathrm{n}+1}\right)=\mathrm{m} \operatorname{vol}(\mathrm{W}) \text { temos: }
$$




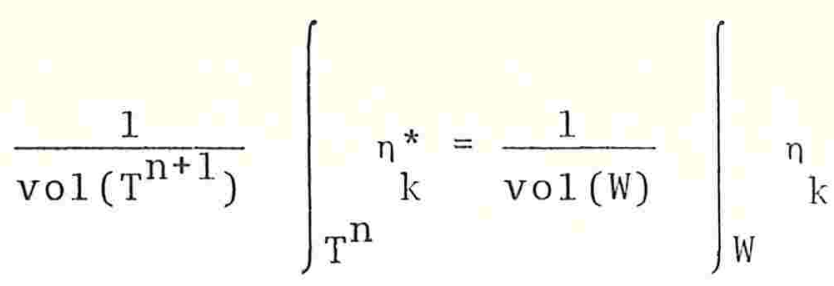

Assim, basta provar a fórmula (2) para uma folheação do toro $\mathrm{T}^{\mathrm{n}+1}$ de curvatura 0 .

Daqui até o final do $\$ 2$, F serā uma folheação de codimensão 1 de $T^{n+1} \subset \mathbb{R}^{2 n+2} ; T^{n+1}=\frac{S^{1} \times S^{1} \times \cdots \times S^{1}}{n+1} ; S^{1} \in \mathbb{R}^{2}$.

Proposição 2.1.1

"Com as notações atē aqui fixadas temos:

$\int_{\mathrm{T}}^{\mathrm{n}+1}{ }^{\mathrm{n}} \mathrm{k}=0 \quad \forall \mathrm{k} \quad$ "

Demonstração:

Seja $\psi_{t}: \mathrm{T}^{\mathrm{n}+1} \longrightarrow \mathrm{T}^{\mathrm{n}+1}$ dada por

$\psi_{t}\left(\rho(x)=\rho(x+t \vec{n})=\exp _{x}(t \vec{N})\right.$

onde

$\rho: \mathbb{R}^{\mathrm{n}+1} \rightarrow \frac{\mathbb{R}^{\mathrm{n}+1}}{\mathbb{Z}^{\mathrm{n}+1}}$

é a projeção natural de $\mathbb{R}^{\mathrm{n}+1}$ sôbre $\mathrm{T}^{\mathrm{n}+1} ; \overrightarrow{\mathrm{N}}(\mathrm{x})$ è um campo vetorial unitärio normal a $F$ definido em toda variedade $\mathrm{T}^{\mathrm{n}+1}$ e determinado pela orientação transversal de $F ; \vec{n}=\rho *(\vec{N})$ esta definido em $\mathbb{R}^{\mathrm{n+1}}$;

; exp denota a aplicação exponencial $\exp _{X}: U \in T_{x} T^{n+1} \rightarrow T^{n+1}$ no 
ponto $\mathrm{x}$.

Para $|t|<\delta, \delta$ suficientemente pequeno, $\psi_{t} \vec{e}$ um difeomorfismo. Alëm disso, temos: $0<\operatorname{det} \psi_{t}^{\prime}=\sum_{i=0}^{n} n_{i} t^{i}$. Pois:

$$
\begin{aligned}
& \operatorname{det} \psi_{t}^{\prime}(x)=\operatorname{det}\left(\operatorname{Id}_{T_{x} T^{n+1}}+\operatorname{tdN}(x)\right) e \\
& \text { det } \psi_{t}^{\prime}(x)>0 \text { para }|t|<\delta
\end{aligned}
$$

\section{Então}

$$
\int_{T}^{n+1} \operatorname{det} \psi_{t}^{\prime}=\sum_{i=0}^{n} t^{i} \int_{T}^{n+1} \sigma_{i}(x)=\operatorname{vol}\left(T^{n+1}\right)
$$

$\operatorname{com}|t|<\delta$

logo, por identidade de polinômios vem:

$$
\int_{\mathrm{T}} \mathrm{n}+1
$$$$
\text { e } \sigma_{0}(x)=\operatorname{vol}\left(T^{n+1}\right)
$$

Isto nos restitui as förmulas (1) e (2) para o caso $\mathrm{W}^{\mathrm{n}+1}=\mathrm{T}^{\mathrm{n}+1}$.

$$
\text { r. d. }
$$

\section{$2.2-0$ caso $c>0$}

Dada uma variedade riemaniana $\mathrm{w}^{\mathrm{n}+1}$ compacta, conexa, sem bordo de curvatura seccional constante positiva c e dimensão $n+1$, sabe-se que ela ê isomêtrica ao quociente da es

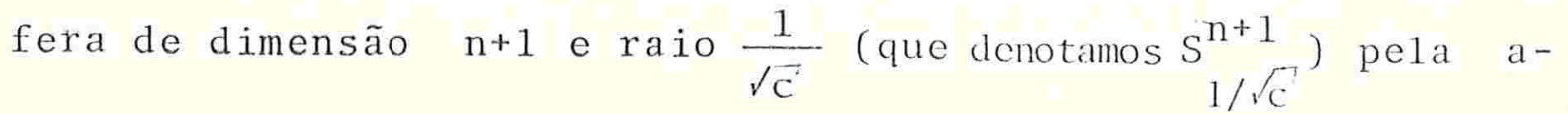


ção de um subgrupo finito de $0(n+1),\left(S 0(n+1)\right.$ se $W^{n+1}$ è orientầe1).

Um argumento anälogo ao apresentado em 2.1 nos mos tra que para demonstrar as fórmulas (1), (2) e (3) basta supor $W^{n+1}=\frac{S^{n+1}}{\sqrt{c}}$

Porëm, uma homotetia na esfera ${ }^{S} \frac{1}{\sqrt{\mathrm{C}}}$ nos mostra que as fórmulas (1), (2) e (3) serão välidas para $\mathrm{S}_{\underline{1}}^{\mathrm{n}+1}$ e se somente se forem vảlidas para $S_{1}^{n+1}=S^{n+1}$

Podemos então supor $W^{n+1}=S^{n+1}$

Seja F uma folheação de codimensão 1 de $S^{n+1}$ trans versalmente orientävel. A existência de uma tal folheação implica que $n+1$ é impar pois $F$ não tem singularidades. A orientação transversal de $F$ nos permite definir um campo de vetores $\vec{n}$ normal a $F$ em cada ponto e globalmente definido em $S^{n+1}$.

Teremos:

$\vec{n}(x) \varepsilon T_{X} S^{n+1} ;|\vec{n}|=1$ e $\vec{n} \perp F$

Seja

$$
\begin{aligned}
& \psi_{t}: S^{n+1} \rightarrow \mathbb{R}^{n+2} \text { a aplicação dada por } \\
& \psi_{t}(x)=x+t \vec{n}(x)
\end{aligned}
$$

Para $t$ suficientemente pequeno, "t $\overrightarrow{\mathrm{c}}$ um difeomor fismo entre $S^{n+1}$ e $\frac{S^{n+1}}{\sqrt{1+t^{2}}}$, ou seja,

se $|t|<\delta$ então $\psi_{t}$ é um difeomorfismo entre a esfera de raio unitärio e a esfera de raio $\sqrt{1+t^{2}}$.

$2.2 .1-\operatorname{Proposição:~}$

"det $\psi_{t}^{\prime}=\sqrt{1+t^{2}} \sum_{i=0}^{n} \eta_{i} t^{i}$ 
Demonstração:

Recordemos inicialmente que as funções $\eta_{i}$ são defí nidas por

$\operatorname{det}\left(I_{T_{x} M_{x}}+\left.t A_{x}\right|_{T_{x} M_{x}}\right)=\sum_{i=0}^{n} n_{i}(x) t^{i}$

onde:

$M_{X} \bar{e}$ a folha que passa pelo ponto $x$

$\mathrm{T}_{\mathrm{X}} \mathrm{M}_{\mathrm{x}}$ é o espaço tangente à folha no ponto $\mathrm{x}$

$A_{x}: T_{x}{ }^{W} \quad T_{x}{ }^{W}$ e definida por:

$A_{X}(Y)=\nabla_{Y}(\vec{n}), \quad \vec{n} \perp F ; \quad Y \varepsilon T_{X} W ; \quad \nabla$ conexão riemannia$|\vec{n}|=1 \quad$ na de $(W,<>)$

e

folha.

$$
\left.A_{x}\right|_{T_{X} M_{x}} \vec{e} \text { a restrição de } \Lambda_{X} \text { ao espaço tangente à }
$$

Note-se que um argumento de ortogonalidade mostra que $A_{x}$ deixa invariante o subespaço $T_{x} M_{x} \subset T_{x} W$.

Passemos então ao câlculo de $\operatorname{det}\left(\psi_{t}^{\prime}\right)$. Teremos:

$$
\mathrm{T}_{\mathrm{x}} \mathrm{M}_{\mathrm{x}} \subset \mathrm{T}_{\psi(\mathrm{x})}\left(\frac{\mathrm{S}^{\mathrm{n}+1}}{\sqrt{1+\mathrm{t}^{2}}}\right) \text {; pois todo vetor perpendicu }
$$

lar ao mesmo tempo a $x$ e $\vec{n}(x)$ será tambëm perpendicular a $x+t \vec{n}(x)$.

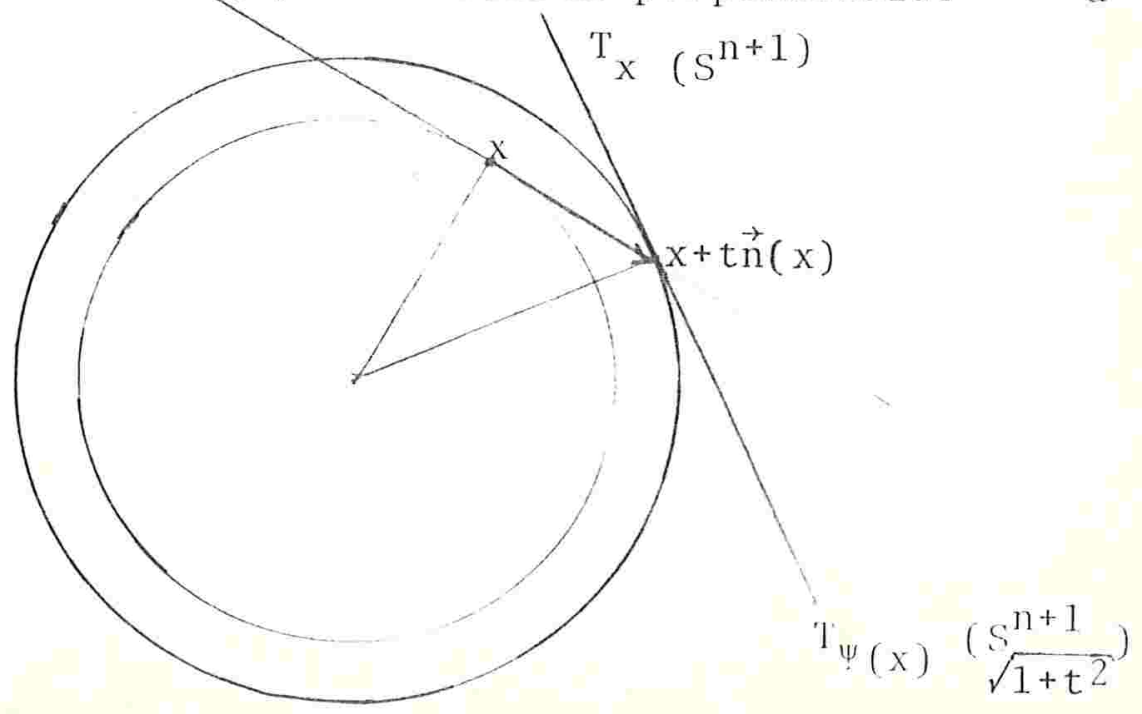


Sejam então dois referenciais ortonormais B e B'10 cais definidos respectivamente sôbre uma vizinhança $U$ de $x$ e $\psi_{t}(U)$ de $\psi_{t}(x)$ satisfazendo:

$$
\begin{aligned}
& B^{\prime}=\left\{e_{1}(x), e_{2}(x), \ldots, e_{n}(x), \vec{n}(x)\right\} \\
& B^{\prime}=\left\{\bar{e}_{1}(\psi(x)), \bar{e}_{2}(\psi(x)), \ldots, \bar{e}_{n}(\psi(x)), \vec{u}(\psi(x))\right\}
\end{aligned}
$$

onde

$$
e_{i}(x) \in T_{x} M_{x} \quad, \quad \bar{e}_{i}\left(\psi_{t}(x)\right)=e_{i}(x) ; 1 \leq i \leq n
$$

e

$$
\vec{u}(x))=\frac{1}{\sqrt{1+t^{2}}} \vec{n}(x)-\frac{t}{\sqrt{1+t^{2}}} x
$$

A motriz jacobiana de $\psi_{t}$ escreve-se:

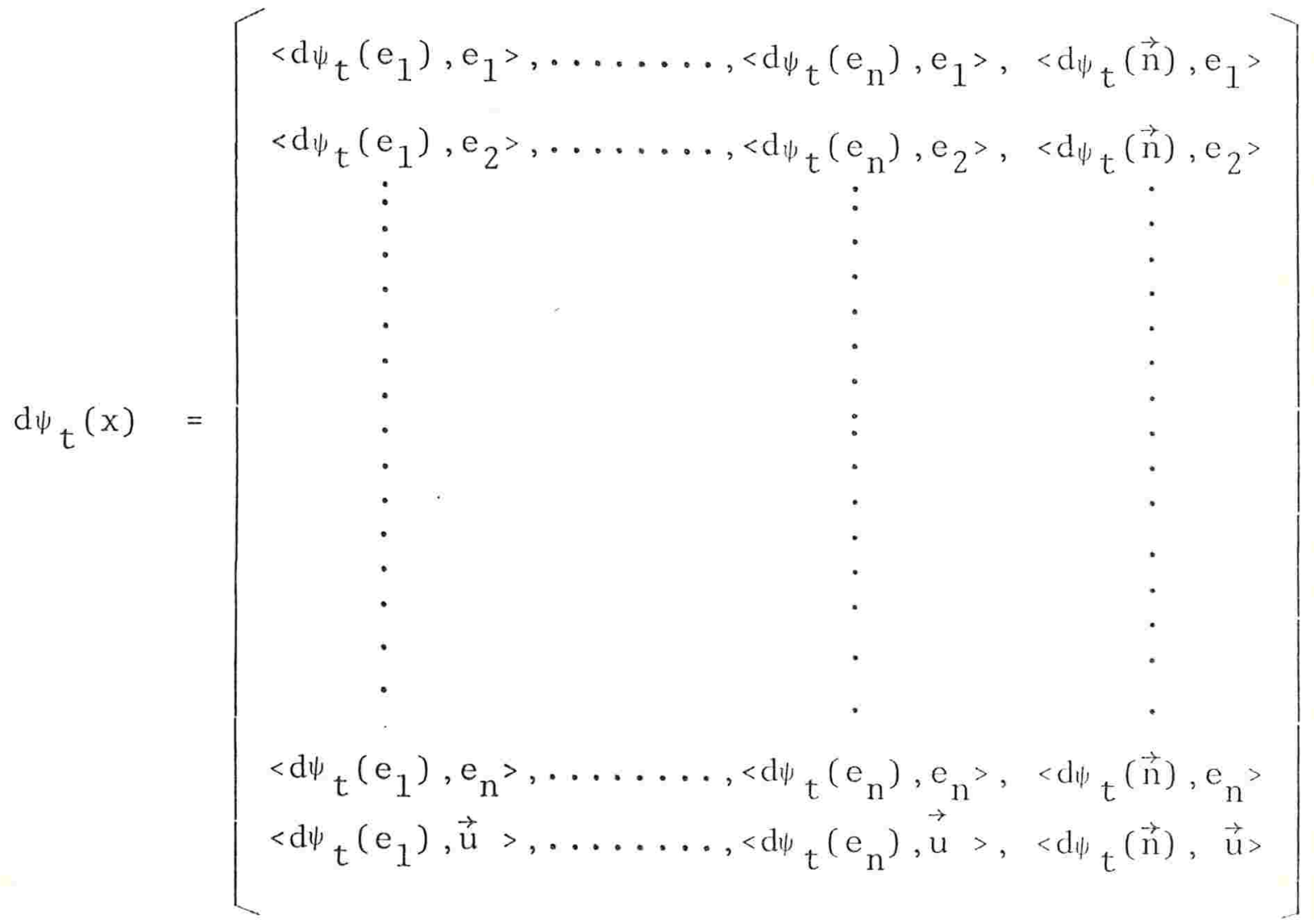


Isto nos dả imediatamente:

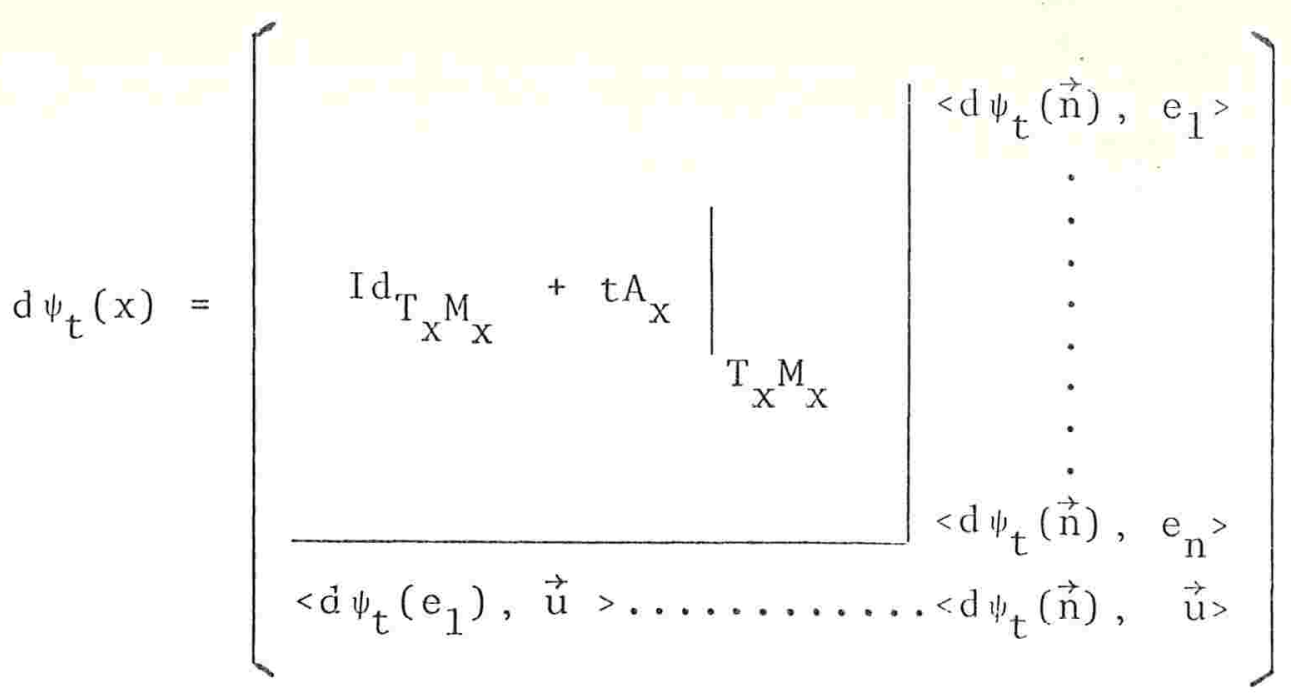

Temos então

$$
\operatorname{det}\left(d \psi_{t}(x)\right)=\sqrt{1+t^{2}} \operatorname{det}\left(\operatorname{Id}_{T_{x} M_{x}}+\left.t A_{x}\right|_{T_{x} M_{x}}\right)
$$

Pois:

1) $<\mathrm{d} \psi_{t}\left(\mathrm{e}_{\mathrm{i}}\right), \overrightarrow{\mathrm{u}}>=0 \quad 1 \leq \mathrm{i} \leq \mathrm{n}$

2) $\left.<d \psi_{t}(\vec{n}), \quad \vec{u}\right\rangle=\left\langle(I+t d \vec{n})(\vec{n}), \frac{1}{\sqrt{1+t^{2}}} \vec{n}-\frac{t}{\sqrt{1+t^{2}}} x\right\rangle=$ $=\frac{1}{\sqrt{1+t^{2}}}-\frac{t^{2}}{\sqrt{1+t^{2}}}<d \vec{n}(\vec{n}), x>=$

Da maneira como foram definidos os $n_{i}$, 'Teremos:

$\operatorname{det}\left(d \psi_{t}(x)\right)=\sqrt{1+t^{2}} \sum_{i=1}^{n} n_{i} t^{i}$ C.C...

O volume da esfera de dimensão impar e raio $\sqrt{1}+t^{2}$ $\vec{e}$ um polinômio de grau impar em $y=\sqrt{1+t^{2}}$

Pelo teorema de mudança de variảveis teremos: 
$\operatorname{vol}\left(\frac{S^{n+1}}{\sqrt{1+t^{2}}}\right)=\left|\int_{S^{n+1}} \operatorname{det} d \psi t\right|$

Para $t$ suficientemente pequeno temos

$\operatorname{det} \mathrm{d}_{t}>0$

Logo, como n+1 ë ímpar temos (Ver [9])

$\int_{S^{n+1}} \operatorname{detd} \psi_{t}=\frac{2 \pi^{\frac{n}{2}}+1}{\left(\frac{n}{2}\right) !}\left({\sqrt{1+t^{2}}}^{n+1}\right.$

Logo ,

$\int_{S^{n+1}} n_{i} t^{i}=\frac{2 \pi^{\frac{n}{2}+1}}{\left(\frac{n}{2}\right) !}\left(\sqrt{1+t^{2}}\right)^{n}$

Portanto

$\frac{1}{\operatorname{vol}\left(S^{n+1}\right)}\left(\int_{S^{n+1}} n_{i}\right) t^{i}=\left(1+t^{2}\right)^{\frac{n}{2}}$

Como a última igualdade é vảlida para qualquer $|t|<\delta$, $t$ positivo temos, aplicando o príncipio de identidade de poli nômios :

$$
\left\{\begin{array}{l}
\frac{1}{\operatorname{vol}\left(S^{n+1}\right)} \int_{S^{n+1}} n_{i}=0 \text { se i é impar } \\
\frac{1}{\operatorname{vol}\left(S^{n+1}\right)} \int_{S^{n+1}}^{n_{i}=}\left(_{\frac{i}{2}}^{\frac{n}{2}}\right. \text { se i é par }
\end{array}\right.
$$


Is to também nos restituirá as fórmulas (1) e (3), uma vez que as funções $\gamma_{2 k}$ são combinações 1 ineares das funções $n_{2 k}$ onde os coeficientes só dependem das dimensões envolvidas e da curvatura seccional constante $\underline{c}$ do espaço em questão. 


\section{$2.3-0$ caso $c<0$}

Seja $<$, > a pseudo - métrica definida em $\mathbb{R}^{n+1}$ por $<x, y>=x_{1} y+x_{2} y_{2}+\ldots+x_{n} y_{n}-x_{n+1} y_{n+1}$

onde $x=\left(x_{1}, x_{2}, \ldots, x_{n+1}\right), y=\left(y_{1}, y_{2}, \ldots, y_{n+1}\right)$

Seja $c<0$ e

$H^{n}(c)=\left\{p \varepsilon \mathbb{R}^{n+1}, p=\left(p_{1}, p_{2}, \ldots, p_{n+1}\right) ; p_{n+1}>0 ;\right.$

$\left.;\langle\mathrm{p}, \mathrm{p}\rangle=\frac{1}{\mathrm{c}}\right\}$

As proposições seguintes são verdadeiras:

1) A pseudo-métrica <, > torna-se uma mêtrica rieman niana quando restrita a $\mathrm{H}^{\mathrm{n}}(\mathrm{c})$.

2) A curvatura seccional de $H^{n}(c)$ nesta mëtrica è constante e igua1 a c.

Estes fatos podem ser vistos em $[10]$.

$H^{n}(c)$ è chamadó "espaço hiperbólico de dimensão n e curvatura c", quando munido das métricas acima.

$$
\begin{aligned}
& \text { Seja } c=-1 \quad n=3 \\
& H^{3}(c)=\left\{(x, y, z) \quad \varepsilon \mathbb{R}^{3}, z>0 ; x^{2}+y^{2}-z^{2}=-1\right\}
\end{aligned}
$$




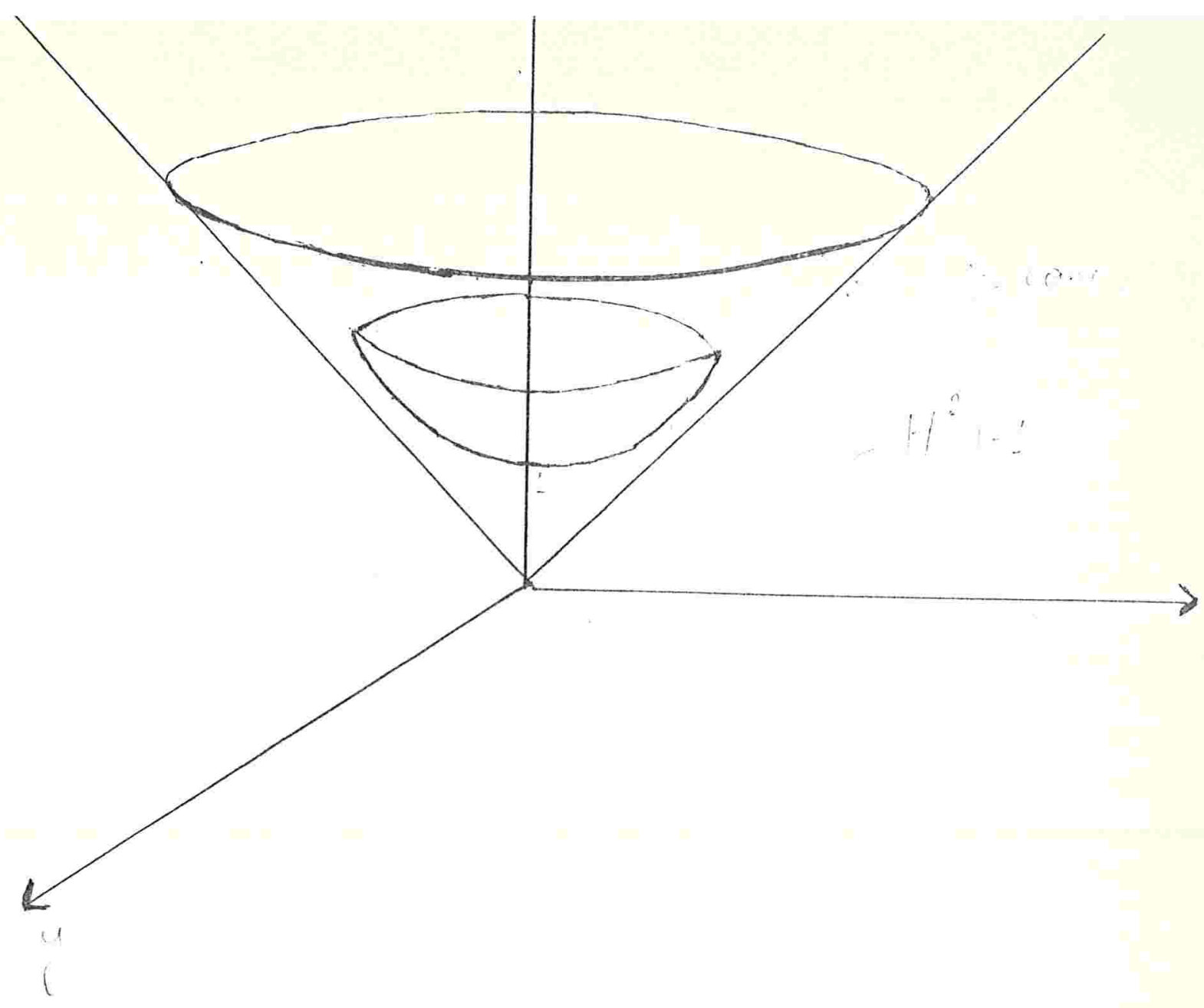

$\mathrm{H}^{\mathrm{n}}=\mathrm{H}^{\mathrm{n}}(-1)$ munido da métrica hiperbölica serä de nominado "espaço hiperböíico standard de dimensão n".

Seja $\vec{n}$ um campo de vetores unitärio (com a métrica hiperbölica) em $\mathrm{H}^{\mathrm{n}}$.

Seja $t>0 \quad e$

$\psi_{t}: H^{n} \rightarrow \mathbb{R}^{n+1}$ dada por

$\psi_{t}(x)=x+t \vec{n}$

\section{3 .1 - Proposição}

$" \psi_{t}(x) \varepsilon H^{n}\left(-1+t^{2}\right), \quad \forall x \varepsilon H^{n}$ " 


\section{Demonstração:}

Queremos provar que:

$<\psi_{t}(x), \psi_{t}(x)>=-1+t^{2}$

Mas sabemos que $\mathrm{H}^{\mathrm{n}}=\mathrm{f}^{-1}(-1)$ onde

$f: \mathbb{R}^{\mathrm{n}+1} \rightarrow \mathbb{R}^{\mathrm{n}+1}$ é dada por

$f\left(x_{1}, x_{2}, \ldots, x_{n+1}\right)=\sum_{i=1}^{n} x_{i}^{2}-x_{n+1}^{2}$

Neste caso, como $\vec{n}$ e vetor tangente de $H^{n}$, temos:

$\vec{n}(x) \cdot \operatorname{grad} f(x)=0, \quad V x \in H^{n}$, onde " . "denota

o produto escalar usual de $\mathbb{R}^{n+1}$. Isto significa que

$\sum_{i=1}^{n} x_{i} \lambda_{i}(x)-x_{n+1} \lambda_{n+1}(x)=0 \quad$, ou equivalentemente,

$x_{n+1} \lambda_{n+1}(x)=\sum_{i=1}^{n} x_{i} \lambda_{i}(x)$, onde $\vec{n}=\left(\lambda_{1}, \lambda_{2}, \ldots, \lambda_{n+1}\right)$

Mas agora, teremos:

$\left\langle\psi_{t}(x), \psi_{t}(x)\right\rangle^{\prime}=\langle x+t \vec{n}(x), x+t \vec{n}(x)\rangle=$

$\left.=\langle x, x\rangle+t^{2}\langle\vec{n}, \vec{n}\rangle(x)+2 t<x, \vec{n}(x)\right\rangle=$

$=-1+t^{2}+2 t<x, \vec{n}(x)>$.

Mas,

$<x, \vec{n}(x)>=\sum_{i=1}^{n} x_{i} \lambda_{i}(x)-x_{n+1} \lambda_{n+1}(x)=0$

$\log 0<\psi_{t}(x), \psi_{t}(x)>=-1+t^{2} \quad c \cdot q \cdot d$. 
Seja $W^{n+1}$ uma variedade riemanniana compacta sem bordo de curvatura seccional constante igual a -1 .

Sabe-se que $\mathrm{W}^{\mathrm{n}+1}$ é isométrica ao quociente de $\mathrm{H}^{\mathrm{n}+1}$ pela ação totalmente discontínua de um subgrupo de isometrias de $H^{n+1}$.

Seja $w_{-1+t^{2}}^{n+1}$ uma variedade riemanniana homotética a $w^{n+1}$ e de curvatura seccional constante igual a $-1+t^{2}$.

$$
\begin{aligned}
\text { Sejam } \Psi_{1}: H^{n+1} \rightarrow \frac{H^{n+1}}{F_{1}}=W^{n+1} & \\
& \begin{array}{c}
n+1 \\
\Psi_{2}: H\left(-1+t^{2}\right) \rightarrow W_{-1+t^{n+1}}^{n+1}
\end{array}
\end{aligned}
$$

as respectivas aplicações quocientes sendo que $F_{1}$ e $F_{2}$ são

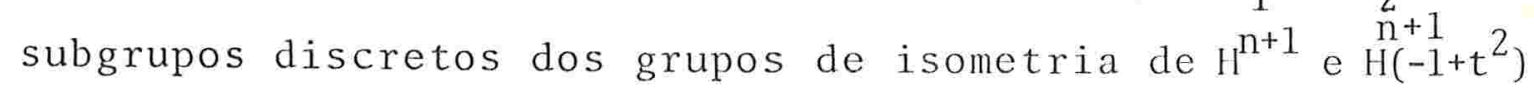
respectivamente. E claro que $\mathrm{F}_{1}$ é isoformo a $\mathrm{F}_{2}$.

Se $\vec{N}$ é um campo vetorial unitärio sobre $w^{n+1}$, se ja $\vec{n}=\Psi_{1}^{*}(N)$ o campo unitário em $H^{n+1}$ correspondente a $\vec{N}$.

\section{3 .2 - Proposição}

"A aplicação $\psi_{t}: H\left(-1+t^{2}\right)$ definida por $\psi_{t}(x)=x+t \vec{n}(x)$ passa ao quociente e induz uma aplicação $\bar{\psi}_{t}: w_{-1+t}^{n+1}$ definida por: $\bar{\psi}_{t}\left(\Psi_{1}(\mathrm{x})\right)=\Psi_{2}\left(\psi_{t}(\mathrm{x})\right)^{\prime \prime}$.

\section{Demonstração}

$$
\text { Sejam } x, x^{\prime} \varepsilon H^{n} \text { tais que } \psi_{1}(x)=\psi_{1}\left(x^{\prime}\right)
$$

Devemos provar que $\psi_{2}\left(\psi_{t}(x)\right)=\psi_{2}\left(\psi_{t}\left(x^{\prime}\right)\right.$

Sabemos que o grupo de isometrias de $\mathrm{H}^{\mathrm{n}+1}$ (bem co mo o de $H\left(-i+t^{2}\right)$ ) è a restrição a $H^{n+1} \quad$ (respectivamente $H\left(=1+t^{2}\right)$ do subgrupo do grupo 1 inear $G L(n+2, \mathbb{R})$ que pre 
serva a pseudo mëtrica $<>$. Chamemos tal subgrupo de L. $(\operatorname{LcGL}(n+2, \mathbb{R}))$. Isto se dá pe1o fato que $H^{n+1}\left(\operatorname{resp} H\left(-1+t^{2}\right)\right)$ são "bolas não degeneradas centradas na origem". (Com respeito a $<>$ ). Sendo assim, $F_{1}$ e $F_{2}$ são restrições de um mesmo sub grupo FcL respectivamente a $\mathrm{H}^{\mathrm{n}+1}$ e $\mathrm{H}\left(-1+\mathrm{t}^{2}\right)$.

Seja oEF. Como F ê um grupo de matrizes temos:

$\sigma\left(\psi_{t}(x)=\sigma(x+t \vec{n}(x))=\sigma(x)+t \sigma(\vec{n}(x))\right.$.

Como o è linear temos:

$\sigma^{\prime}(x) \cdot \vec{n}(x)=\sigma(\vec{n}(x))$ pois $\sigma^{\prime}(x)=\sigma$

então

$\sigma(x)+\operatorname{to}(\vec{n}(x))=\sigma(x)+t \sigma^{\prime}(x) \cdot \vec{n}(x)$.

Assim, para algum elemento $\sigma E F$ teremos:

$\psi_{2}\left(\psi_{t}\left(x^{\prime}\right)\right)=\psi_{2}\left(\psi_{t}(\sigma(x))=\psi_{2}\left(\sigma(x)+t \sigma^{\prime}(x) \cdot \vec{n}(x)\right)=\right.$

$=\Psi_{2}\left(\sigma\left(\psi_{t}(\mathrm{x})\right)=\Psi_{2}\left(\psi_{t}(\mathrm{x})\right) \quad \mathrm{c} \cdot \mathrm{q} \cdot \mathrm{d}\right.$.

\section{3 .3 - Proposição}

"A aplicação $\bar{\psi}_{t}: W^{n+1} \rightarrow W_{-1+t^{n+1}}^{n}$ associada ao campo vetorial $\vec{N}$ em $W^{n+1}$ satisfaz:

$\operatorname{det}\left(\bar{\psi}_{t}^{\prime}(x)\right)=\sqrt{1-t^{2}} \sum_{i=0}^{n} \eta_{i} t^{i}$ para $0<t<1 "$

\section{Demonstração:}

Seja $\Psi_{1}: H^{n+1} \rightarrow W^{n+1}=\frac{H^{n+1}}{F}$ a aplicação quociente definida anteriormente.

$\Psi_{1}$ é uma isometria local, logo, se $y \in H^{1 n+1}$ então $\operatorname{det}\left(\bar{\psi}_{t}^{\prime}\left(\Psi_{1}(y)\right)=\operatorname{det}\left(\psi_{t}^{\prime}(y)\right)\right.$. 
Mas det $\left(\psi_{t}^{\prime}(y)\right)=\sqrt{1-t^{2}} \sum_{i=0}^{n} n_{i} t^{i} \quad e$

o cảlculo é anālogo ao explicitado na proposição 2.2.1. $c \cdot q \cdot d \cdot$

2.3 .4 - Proposição:

A aplicação $\bar{\psi}_{t}: W^{n+1} \rightarrow W_{-1+t^{2}}^{n+1}$ è um difeomorfismo para t suficientemente pequeno".

\section{Demonstração:}

Existe $\delta>0$ tal que $\bar{\psi}_{t}$ é difeomorfismo local pa ra $|t|<\delta$, pois $\operatorname{det}\left(\bar{\psi}_{t}^{\prime}(x)=\operatorname{det}\left(\psi_{t}^{\prime}(y)\right)\right.$ onde $\Psi(y)=x, \operatorname{det} \Psi^{\prime}(t)$ $\vec{e} \quad$ contínua em $t$ e det $\psi_{0}^{\prime}=1$.

Como $W^{n+1}$ è compacto existe $\delta^{\prime}<\delta$ tal que $\bar{\psi}_{t} \vec{e}$ d $\underline{i}$ feomorfismo para $|t|<\delta^{\prime}$. c. c.d.

Passemos agora à demonstração do teorema principal desse capítulo para o caso onde a curvatura do espaço ambiente $\vec{e}$ constante negativa.

Seja F uma folheação de codimensão 1 sôbre $W^{n+1}$ transversalmente orientada.. $W^{n+1}$ e uma variedade compacta conexa sem bordo e orientável. Seja $c<0$ a curvatura (constante) de $\mathrm{w}^{\mathrm{n}+1}$.

\section{$2.3 .5-$ Teorema}

"Com as notações até aqui introduzidas, $\frac{1}{\operatorname{vol}\left(w^{n+1}\right)} \int_{W^{n+1}}^{n} k=\left(\begin{array}{l}c \frac{k}{2}\left(\begin{array}{l}\frac{n}{2} \\ \frac{k}{2}\end{array}\right) \begin{array}{l}\text { se } n \text { e } k \text { são } \\ \text { pares }\end{array} \\ 0 \text { nos outros casos" }\end{array}\right.$ 


\section{Demonstração:}

Pelas proposições $2.3 .3,2.3 .4$, e pelo teorema de mudança de variações para integrais, teremos:

$$
\sqrt{1-t^{2}} \int_{w^{n+1}} \sum_{i=0}^{n} \eta_{i} t^{i}=\operatorname{vol}\left(w_{-1+t^{2}}^{n+1}\right)
$$

Mas, como $\mathrm{w}_{-1+t^{\mathrm{n}+1}}^{2}$ é homotética a $\mathrm{w}^{\mathrm{n}+1}$ com razão de homotetia $\sqrt{1-t^{2}}$, vale:

$\operatorname{vol}\left(w_{-1+t^{2}}^{n+1}\right)=\left(\sqrt{1-t^{2}}\right)^{n+1} \cdot \operatorname{vol}\left(w^{n+1}\right)$.

Então,

$\frac{1}{\operatorname{vol}\left(W^{n+1}\right)} \sqrt{1-t^{2}} \int_{W} i \sum_{=0}^{n} n_{i} t^{i} \sqrt{\left(1-t^{2}\right)} n+1$

logo,

$$
\frac{1}{\operatorname{vol}\left(w^{n+1}\right)} \quad \sum_{1=0}^{n} n_{i} t^{i}=\left(\sqrt{1-t^{2}}\right)^{n}
$$

Como a igualdade vale $\forall t$, com $|t|<\delta^{\prime}$ e o primeiro membro é um polinômio, o teorema fica demonstrado para o ca so em que $\mathrm{c}=-1$.

O caso geral segue se considerarmos uma homotetia de razão $\sqrt{-c}$. c.q.d. 


\section{CAPITULO II}

"Uma Fórmula e Suas Consequências".

\section{$\S 1$ - Introdução}

Neste capítulo apresentamos alguns resultados con cernentes à geometria integral das folheações para o caso em que a curvatura do espaço ambiente não é constante.

Precisamente, seja $W^{n+1}$ uma variedade riemanniana compacta conexa e sem bordo onde está definida uma folheação transversalmente orientada $F$ de codimensão 1. Trataremos neste capítulo de fornecer relações entre as possíveis integrais de curvatura das folhas e a geometria do espaço ambiente.

Uma primeira questão que surge é a de saber como varia a mẻdia de curvatura das folhas em função de limites im postos à variação do tensor de curvatura de $w^{n+1}$.

Sobre isto, D. Asimov demonstrou o seguinte:

$(\operatorname{ver}[1])$.

Teorema $|A|$ :

"Seja $w^{3}$ variedade riemanniana compacta e sem bor do e $F$ uma folheação de codimensão 1 sôbre $W^{3}$.

Suponhamos que existam constantes $K_{1}, K_{2}$ tais que:

$K_{1} \leqslant c\left(x, Q_{2}\right) \leqslant K_{2} \quad \forall x \in W^{3}, V_{2} \quad c \quad T_{x} w^{3}$

Então

$$
2 K_{1} \leqslant \frac{1}{\operatorname{vol}\left(W^{3}\right)} \int_{W^{3}} K \leqslant 2 K_{2}
$$


onde, $\mathrm{K}: \mathrm{W}^{3} \rightarrow \mathbb{R}$ é a curvatura de Gauss da folha que passa por $x$ no ponto $x$ com a métrica induzida pela inclusão da fó 1ha em $w^{3}$,

, $c\left(x, Q_{2}\right)$ a curvatura seccional de $w^{3}$ no ponto $x$ e na direção do 2 - plano $Q_{2} c T_{x} W^{3}$,

$\operatorname{dim} W^{3}=3^{\prime \prime}$

Este resultado serä generalizado para dimensão qualquer considerando-se como integrando a curvatura escalar das folhas.

Alẻm disso provaremos o seguinte:

1) Se $w^{n+1}$ e uma variedade de Einstein (curvatura de Ricci constante), então a integral da curvatura escalar das folhas não depende da folheação.

2) Se existe um inteiro $q, 1 \leqslant 2 q \leqslant n-1$ tal que as curvaturas $2 q$ - seccionais de $w^{n+1}$ não se anulam então $W^{n+1}$ não admite folheação totalmente geodẹ sica de codimensão 1. Em particular os espaços de curvatura seccional sempre não nula têm esta propriedade.

3) Existem $(n+1)$ - formas $\Xi_{2 k}$ sôbre $W^{n+1}$ (que depen dem da variação do campo de vetores unitärio $N$ que determina a orientação transversal de $F$ e é nor mal a $F$ em cada ponto) que definem classes de co homologia em $\mathrm{H}^{\mathrm{n}+1}\left(\mathrm{~W}^{\mathrm{n+1}}, \mathbb{R}\right)$. 
Precisamente:

$$
\int_{W^{n+1}}{ }^{\Xi_{2 k}}=\int_{W^{n+1}}{ }^{c_{2 k}} \quad \text { não depende da }
$$

folheação $F, k=0,1, \ldots,\left[\frac{\mathrm{n}+1}{2}\right]$,

, onde $c_{2 k}$ è a curvatura $2 k$ - escalar de $w^{n+1}$.

Tal classe de cohomologia só depende da métrica $<>$ de $W^{n+1}$.

\section{§2. Notações:}

Seja $x \in W^{n+1}$, UЭx um aberto de $W^{n+1}$,

, $\left\{e_{1}, e_{2}, \ldots, e_{n}, e_{n+1}\right\}$ um referencial ortonormal adaptado sobre U:

$$
<e_{i}, e_{j}>=\delta_{i j} ; 1<i, j<n+1 ; N=e_{n+1} \perp F \text {. }
$$

o correferencial, as formas de conexão e de curva tura associados são dados por:

$$
\begin{aligned}
& \theta_{i}\left(e_{j}\right)=\delta_{i j}, \delta_{i j}=0 \text { se } i \neq j, \delta_{i i}=1 \\
& \omega_{i j}(u)=\left\langle\nabla_{u}\left(e_{i}\right), e_{j}\right\rangle, \Omega_{i j}=d \omega_{i j}-\sum_{k=1}^{n+1} \omega_{i k} \wedge \omega_{k j}
\end{aligned}
$$

$<,>$ e $\nabla$ denotam respectivamente o produto escalar e a cone xão riemanniana associados a $\left(W^{\mathrm{n}+1},<,>\right)$. 
As fórmulas de estrutura são dadas por

$$
\mathrm{d} \theta_{i}=\sum_{k=1}^{n+1} \omega_{i k} \Lambda \theta_{k} \quad, \quad d \omega_{i j}=\sum_{k=1}^{n+1} \omega_{i k} \wedge \omega_{k j}+\theta_{i j}
$$

E a identidade de Bianchi escreve-se:

$$
\mathrm{d} \Omega_{i j}=\sum_{k=1}^{n+1} \omega_{i k} \Lambda \Omega_{k j}-\Omega_{i k} \Lambda \omega_{k j} .
$$

Esta é a notação utilizada, por exemplo em |3|

$$
\S 3 \text { - As formas } \psi_{\mathrm{k}, 2 \ell, \mathrm{n}}, \phi_{\mathrm{k}, 2 \ell, \mathrm{n},}, \Gamma_{2 \mathrm{r}, \mathrm{n}} \mathrm{e}
$$

um lema combinatório.

Com as notações introduzidas no $\$ 2$ pode-se definir n-formas ${ }^{\Psi} \mathrm{k}, 2 \ell, \mathrm{n}$

$$
0 \leqslant k \leqslant n ; 0 \leqslant 2 \ell \leqslant n ; k+2 \ell \leqslant n
$$

e $(n+1)$ - formas $\phi_{k}, 2 \ell, \quad n$

$$
0 \leqslant k \leqslant n-1 ; 0 \leqslant 2 \ell \leqslant n-2 ; \quad k+2 \ell \leqslant n-1
$$

sôbre um aberto $\mathrm{UcW}^{\mathrm{n}+1}$.

Estas formas estão diretamente associadas à folhea ção $F$ e são definidas através de um referencial ortonormal adaptado $\left\{\mathrm{e}_{1}, \ldots, \mathrm{e}_{\mathrm{n}}, \mathrm{e}_{\mathrm{n}+1}\right\}$ por:

$$
\begin{aligned}
& { }^{\psi} \mathrm{k}, 2 \ell, \mathrm{n}=\sum_{\sigma \varepsilon \mathrm{S}_{\mathrm{n}}} \operatorname{sgn}(\sigma) \omega_{\sigma(1)} \mathrm{n}+1 \wedge \omega_{\sigma(2) \mathrm{n}+1} \wedge \ldots \wedge \\
& \Lambda \omega_{\sigma(k)} n+1 \Lambda \Omega_{\sigma(k+1)} \sigma(k+2) \Lambda \ldots \Lambda \Omega_{\sigma(k+2 \ell-1)} \sigma(k+2 \ell)^{\Lambda} \\
& \Lambda \theta_{\sigma(k+2 \ell+1)} \Lambda \theta_{\sigma(k+2 \ell+2)} \Lambda \ldots \Lambda \quad \theta_{\sigma(n)}
\end{aligned}
$$


$\phi_{\mathrm{k}, 2 \ell, \quad \mathrm{n}}=\sum_{\sigma \varepsilon \mathrm{S}_{\mathrm{n}}} \operatorname{sgn}(\sigma) \Omega_{\sigma(1)} \mathrm{n}+1 \Lambda \kappa_{\sigma(2)} \mathrm{n}+1 \Lambda \omega_{\sigma(3) \mathrm{n}+1} \Lambda$

$\Lambda \ldots . \Lambda \omega_{\sigma(k+1) n+1} \Lambda \Omega_{\sigma(k+2) \sigma(k+3)}{ }^{\Lambda} \Omega_{\sigma(k+4) \sigma(k+5)} \Lambda$

$\Lambda \ldots . \Lambda \Omega_{\sigma(k+2 \ell) \sigma(k+2 \ell+1)}{ }^{\Lambda \theta_{\sigma(k+2 \ell+2)}} \quad \Lambda \theta_{\sigma(k+2 \ell+3)} \Lambda \ldots \ldots \Lambda$

$\Lambda \theta_{\sigma(\mathrm{n})} \cdot$

onde $S_{n}$ denota o grupo simêtrico de permutações de $n$ elementos.

\section{$3.1-\underline{\text { Lema }}$}

"As formas $\Psi_{\mathrm{k}, 2 \ell, \mathrm{n}}, \phi_{\mathrm{k}, 2 \ell, \mathrm{n}}$ são globais em W"

\section{Demonstração}

Seja $\left\{\overline{\mathrm{e}}_{1}, \overline{\mathrm{e}}_{2}, \ldots \ldots, \overline{\mathrm{e}}_{\mathrm{n}}, \overline{\mathrm{e}}_{\mathrm{n}+1}\right\}$ um outro referencial or tonormal adaptado a F. Tem-se:

$$
\begin{aligned}
& \bar{e}_{i}=\sum_{j=1}^{n} a_{i j} e_{j} ; 1<i<n ; \\
& \bar{e}_{n+1}=e_{n+1} ; \operatorname{det}\left(a_{i j}\right)=1 ;\left(a_{i j}\right) \text { é uma matriz orto- }
\end{aligned}
$$

gona 1 . 
As "novas" formas de conexão e curvatura relacio nam-se com as "antigas" por:

$$
\begin{aligned}
& \bar{\omega}_{i n+1}=\sum_{j=1}^{n} a_{i j} \omega_{j n+1} \\
& \bar{\Omega}_{i j}=r, \sum_{j=1}^{n} a_{i r} a_{j s} \Omega_{r s} \\
& \bar{\Omega}_{i n+1}=\sum_{j=1}^{n} a_{i j} \Omega_{j} n+1 \\
& 1 \leqslant i, j \leqslant n
\end{aligned}
$$

Então,

$$
\begin{aligned}
& \bar{\Psi}_{\mathrm{k}, 2 \ell, \mathrm{n}}=\sum_{\sigma \varepsilon S_{\mathrm{n}}} \operatorname{sgn}(\sigma)\left(\sum_{\mathrm{r}=1}^{\mathrm{n}} \mathrm{a}_{\sigma(1) \mathrm{r}} \omega_{\mathrm{r} n+1}\right) \Lambda \\
& \Lambda\left(\sum_{\mathrm{r}=1}^{\mathrm{n}} \mathrm{a}_{\sigma(2) \mathrm{r}} \omega_{\mathrm{r} \mathrm{n}+1}\right) \Lambda \ldots \Lambda\left(\sum_{\mathrm{r}=1}^{\mathrm{n}} \mathrm{a}_{\sigma(\mathrm{k}) \mathrm{r}}{ }^{\omega_{\mathrm{r} n+1}}\right) \Lambda
\end{aligned}
$$$$
\Lambda\left(\sum_{r, s=1}^{n} a_{\sigma(k+1) r} a_{\sigma(k+2) s}{ }^{\Omega} r s\right) \Lambda\left(\sum_{r, s=1}^{n} a_{\sigma(k+3) r} a_{\sigma(k+4) s} \Omega_{r s}\right) \Lambda
$$$$
\Lambda \ldots \Lambda\left({ }_{\mathrm{r}, \mathrm{s}=1}^{\mathrm{n}} \mathrm{a}_{\sigma(\mathrm{k}+2 \ell-1) r} \mathrm{a}_{\sigma(\mathrm{k}+2 \ell) \mathrm{s}}{ }_{\mathrm{rs}}\right) \Lambda
$$

$\Lambda\left(\sum_{\mathrm{r}=1}^{\mathrm{n}} \mathrm{a}_{\sigma(\mathrm{k}+2 \ell+1) r} \theta_{\mathrm{r}}\right) \Lambda\left(\sum_{\mathrm{r}=1}^{\mathrm{n}} \mathrm{a}_{\sigma(\mathrm{k}+2 \ell+2) r} \theta_{\mathrm{r}}\right) \Lambda \ldots \Lambda$

$$
\Lambda\left(\begin{array}{ccc}
\sum_{r=1}^{n} & a_{\sigma(n) r} & \theta_{r}
\end{array}\right)
$$

A simetria de $S_{n}$ nos dá imediatamente:

$$
\begin{aligned}
& \bar{\Psi}_{\mathrm{k}, 2 \ell, \mathrm{n}}=\sum_{\sigma \varepsilon \mathrm{S}_{\mathrm{n}}} \sum_{\tau \varepsilon S_{\mathrm{n}}} \operatorname{sgn}(\sigma) \operatorname{sgn}(\tau) \mathrm{a}_{\sigma(1) \tau(1)}{ }_{\tau(1) \mathrm{n}+1}^{\omega} \Lambda \\
& \Lambda \mathrm{a}_{\sigma(2) \tau(2)}{ }^{\omega_{\tau(2)} \mathrm{n}+1} \wedge \ldots \Lambda \mathrm{a}_{\sigma(\mathrm{k}) \tau(\mathrm{k})}{ }^{(1)} \tau(\mathrm{k}) \mathrm{n}+1
\end{aligned}
$$




$$
\begin{aligned}
& \Lambda \mathrm{a}_{\sigma(\mathrm{k}+1)} \tau(\mathrm{k}+1) \mathrm{a}_{\sigma(\mathrm{k}+2)} \tau(\mathrm{k}+2) \cdot \Omega_{\tau(k+1)} \tau(\mathrm{k}+2) \Lambda \\
& \Lambda a_{\sigma(k+3) \tau(k+3)} a_{\sigma(k+4)} \tau(k+4) \Omega_{\tau(k+3)} \tau(k+4) \Lambda \ldots \ldots \Lambda \\
& \Lambda \mathrm{a}_{\sigma(k+2 \ell-1) \tau(k+2 \ell-1)} \mathrm{a}_{\sigma(k+2 \ell) \tau(k+2 \ell)}{ }^{\Omega_{\tau}} \tau(\mathrm{k}+2 \ell-1) \tau(k+2 \ell) \quad \Lambda \\
& \Lambda \mathrm{a}_{\sigma(\mathrm{k}+2 \ell+1) \tau(\mathrm{k}+2 \ell+1)} \theta_{\tau(\mathrm{k}+2 \ell+1)} \Lambda \mathrm{a}_{\sigma(k+2 \ell+2) \tau(k+2 \ell+2)} \theta_{\tau}(\mathrm{k}+2 \ell+2) \Lambda \\
& \Lambda \ldots \ldots . \wedge a_{\sigma(n)} \tau(n) \theta_{\tau}(n)
\end{aligned}
$$

Como $\operatorname{det}\left(a_{i j}\right)=1$ temos

$$
\bar{\Psi}_{\mathrm{k}, 2 \ell, \mathrm{n}}=\Psi_{\mathrm{k}, 2 \ell, \mathrm{n}}
$$

Anàlogamente mostra-se que $\bar{\phi}_{\mathrm{k}, 2 \ell, \mathrm{n}}=\phi_{\mathrm{k}, 2 \ell, \mathrm{n}} \mathrm{c} \cdot \mathrm{q} \cdot \mathrm{c}$.

\section{2 - Lema Fundamenta1}

$"{ }^{\mathrm{d}} \Psi_{\mathrm{k}, 2 \ell, \mathrm{n}}={ }^{\mathrm{k}} \phi_{\mathrm{k}-1,2 \ell, \mathrm{n}}+2 \ell \phi_{\mathrm{k}+1,2(\ell-1), \mathrm{n}}-$

$-(1)^{\mathrm{n}}(\mathrm{n}-\mathrm{k}-2 \ell){ }_{\mathrm{k}+1,2 \ell, \mathrm{n}}{ }^{\Lambda} \theta_{\mathrm{n}+1}$ se k é impar "

\section{Demonstração:}

Façamos $\mathrm{d}_{\mathrm{k}, 2 \ell, \mathrm{n}}=\mathrm{A}+\mathrm{B}+\mathrm{C}$, onde:

$\mathrm{A}=\mathrm{k} \sum_{\sigma \varepsilon \mathrm{S}_{\mathrm{n}}} \operatorname{sgn}(\sigma) \mathrm{d} \omega_{\sigma(1) \mathrm{n}+1} \Lambda \omega_{\sigma(2) n+1} \Lambda \ldots \omega_{\sigma(\mathrm{k}) n+1} \Lambda$ $\Lambda \Omega_{\sigma(\mathrm{k}+1)} \sigma(\mathrm{k}+2) \Lambda \ldots \Lambda \Omega_{\sigma(\mathrm{k}+2 \ell-1)} \sigma(\mathrm{k}+2 \ell){ }^{\Lambda} \theta_{\sigma(\mathrm{k}+2 \ell+1)} \Lambda \ldots . \Lambda$ $\Lambda \theta_{\sigma(n)} \cdot$ 
$\mathrm{B}=(-1)^{\mathrm{k}} \ell \sum_{\sigma \varepsilon \mathrm{S}_{\mathrm{n}}} \operatorname{sgn}(\sigma) \omega_{\sigma(1) n+1} \Lambda \omega_{\sigma(2)} \mathrm{n+1} \Lambda \ldots \Lambda \omega_{\sigma(\mathrm{k}) \mathrm{n}+1} \Lambda$

$\Lambda \mathrm{d} \Omega_{\sigma(k+1)} \sigma(k+2) \Lambda \Omega_{\sigma}(k+3) \sigma(k+4) \Lambda \ldots \Lambda \Omega_{\sigma}(k+2 \ell-1) \sigma(k+2 \ell) \Lambda$

$\Lambda \theta_{\sigma(\mathrm{k}+2 \ell+1)} \Lambda \ldots \Lambda \theta_{\sigma(\mathrm{n})}$

$C=(-1)^{k}(n-k-2 \ell) \sum_{\sigma \varepsilon S_{n}} \operatorname{sgn}(\sigma) \omega_{\sigma(1) n+1} \ldots \Lambda \omega \sigma(k) n+1^{\Lambda \Omega} \sigma(k+1) \sigma(k+2)^{\Lambda \ldots \Lambda}$

$\Lambda \Omega_{\sigma(\mathrm{k}+2 \ell-1)} \sigma(\mathrm{k}+2 \ell){ }^{\Lambda \mathrm{d} \theta_{\sigma(\mathrm{k}+2 \ell+1)}} \quad \Lambda \ldots . \theta_{\sigma(\mathrm{n})}$.

Teremos :

$\mathrm{A}=\mathrm{A}_{1}+\mathrm{k} \phi_{\mathrm{k}-1,2 \ell, \mathrm{n}}$, onde

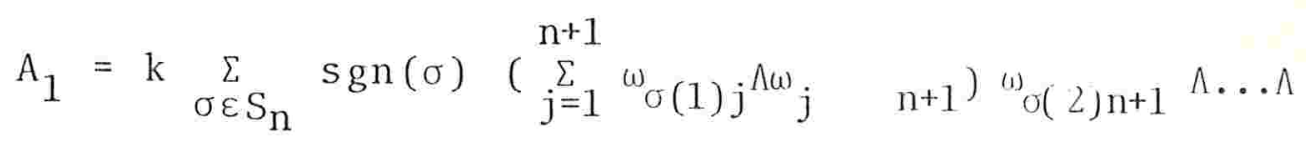

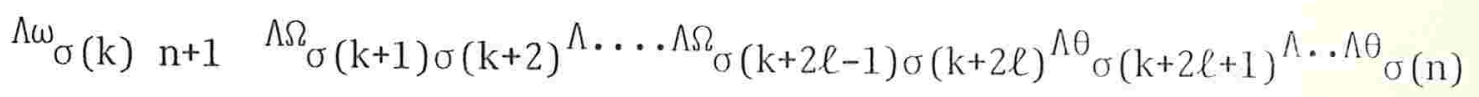

$\mathrm{A}_{1}$ decompõe-se claramente em duas partes:

$$
\begin{aligned}
& A_{1}=A_{11}+A_{12} \text { onde } \\
& A_{11}=(-1)^{\mathrm{k}+1} \quad 2 \ell k \sum_{\sigma \varepsilon S_{n}} \operatorname{sgn}(\sigma) \omega_{\sigma(1)} n+1 \wedge \ldots \Lambda \omega_{\sigma(k) n+1} \Lambda
\end{aligned}
$$

$\Lambda \omega_{\sigma(k+1)} \sigma(1){ }_{\sigma(1)}^{\Lambda} \sigma(\mathrm{k}+2){ }^{\Lambda} \Omega_{\sigma(\mathrm{k}+3) \sigma(\mathrm{k}+4)}{ }^{\Lambda} \ldots \Lambda \Omega_{\sigma(\mathrm{k}+2 \ell-1) \sigma(\mathrm{k}+2 \ell)} \Lambda$

$\Lambda \theta_{\sigma(k+2 \ell+1)} \Lambda \ldots \Lambda \theta_{\sigma(n)}$

$A_{12}=(-1)^{k+1}(n-k-2 \ell) k \sum_{\sigma \varepsilon S_{n}} \operatorname{sgn}(\sigma) \omega_{\sigma(1) n+1} \wedge \ldots \wedge \omega_{\sigma(k) n+1} \Lambda$

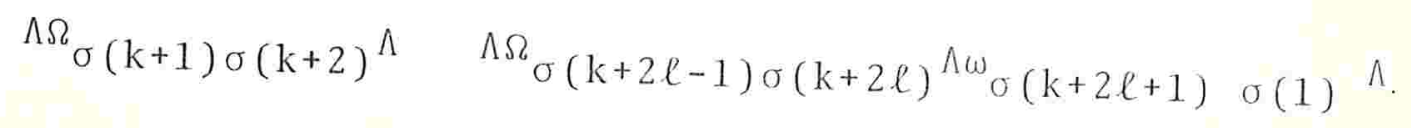

$\Lambda \theta_{\sigma(1)} \Lambda \theta_{\sigma(k+2 \ell+2)} \Lambda \ldots . \Lambda \theta_{\sigma(n)}$

Por outro 1ado, temos: 


$$
\begin{aligned}
& \mathrm{B}=-\mathrm{A}_{11}+\mathrm{B}_{1}+2 \ell \phi_{\mathrm{k}+1}, 2(\ell-1), \mathrm{n} \quad \text { onde } \\
& B_{1}=-2 \ell(-1)^{k}(n-k-2 \ell) \sum_{\sigma \varepsilon S_{n}} \operatorname{sgn}(\sigma) \omega_{\sigma(1) n+1} \Lambda \ldots \Lambda \omega_{\sigma(k) n+1} \Lambda
\end{aligned}
$$

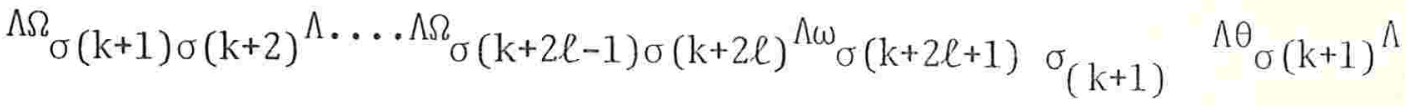

$$
\begin{aligned}
& \Lambda \theta_{\sigma(k+2 \ell+2)} \Lambda \ldots \Lambda \theta_{\sigma(n)}
\end{aligned}
$$

No cálculo da parcela C encontramos:

$\mathrm{C}=-\mathrm{A}_{12}-\mathrm{B}_{1}-(-1)^{\mathrm{n}}(\mathrm{n}-\mathrm{k}-2 \ell) \psi_{\mathrm{k}+1,}, 2 \ell, \mathrm{n} \quad \Lambda \theta_{\mathrm{n}+1}$

Logo

$$
\begin{aligned}
& { }^{\mathrm{d}} \Psi_{\mathrm{k}, 2 \ell, \mathrm{n}}=\mathrm{A}+\mathrm{B}+\mathrm{C}=\mathrm{k} \phi_{\mathrm{k}-1,2 \ell, \mathrm{n}}+2 \ell \phi_{\mathrm{k}+1,2(\ell-1), \mathrm{n}^{-}} \\
& -(-1)^{\mathrm{n}}(\mathrm{n}-\mathrm{k}-2 \ell){ }_{\mathrm{k}+1,2 \ell, \mathrm{n}} \wedge \theta_{\mathrm{n}+1} \\
& \mathrm{c} \cdot \mathrm{q} \cdot \mathrm{d} .
\end{aligned}
$$

\section{3 - Definição:}

Pode-se associar à folheação $F,(n+1)$ - formas $\Gamma_{2 r, n}$ sôbre $W$ para $r=0,1, \ldots,\left[\frac{n}{2}\right\rfloor$, definidas pela equação:

$$
\begin{aligned}
& \Gamma_{2 r, n}=\eta_{2 r, n} \wedge \theta_{n+1}+ \\
& +(-1)^{r+1} \frac{(2 r-1)(2 r-3) \ldots \ldots 5 \cdot 3}{2 \cdot 4 \cdot 6 \ldots \ldots(2 r-4)(2 r-2)} \phi_{0}, 2 r-2, n
\end{aligned}
$$

onde 


$$
\begin{aligned}
& \eta_{2 r, n}=(n-2 r+1)\left[-\Psi_{2 r, 0, n}+\frac{2 r-1}{2} \Psi_{2 r-2,2, n}-\right. \\
& -\frac{(2 r-1)(2 r-3)}{2.4} \Psi 2 r-4,4, n+\ldots .(-1) \frac{r-1(2 r-1)(2 r-3) \ldots .7}{2.4 .6 \ldots(2 r-6)(2 r-4)} \\
& \left.. \Psi_{4,2 r-4, n+(-1)^{r}} \frac{(2 r-1)(2 r-3) \ldots .7 .3}{2.4 .6 \ldots(2 r-6)(2 r-4)(2 r-2)} \Psi_{2,2 r-2, n}\right] .
\end{aligned}
$$

\section{4 - Corolärio:}

"As formas $\Gamma_{2 \mathrm{r}, \mathrm{n}}$ são exatas" .

Demonstração:

Basta tomar

$$
\begin{aligned}
& \tau_{2 r-1, n}=\Psi_{2 r-1,0, n}-\frac{2 r-1}{2} \Psi_{2 r-3,2, n}+ \\
& +\frac{(2 r-1)(2 r-3)}{2.4} \Psi_{2 r-5,4, n}-\ldots \ldots+ \\
& +(-1)-2 \frac{(2 r-1)(2 r-3) \ldots \ldots(2 r-6)(2 r-4)(2 r-2)}{2.4 \ldots \ldots} \Psi_{2,2 r-2, n}^{r-2}
\end{aligned}
$$

e aplicar o lema fundamental para obter

$$
\mathrm{d}_{2 \mathrm{r}-1, \mathrm{n}}=\Gamma_{2 \mathrm{r}-\mathrm{n}} \mathrm{c} \cdot \mathrm{q} \cdot \mathrm{d} \text {. }
$$

$\S 4$ - Os Resu1tados

4.1 - Teorema:

"Se as curvaturas seccionais de W são limitadas por $K_{1}$ e $K_{2}$, ou seja $K_{1} \leqslant c\left(x, Q_{2}\right) \leqslant K_{2}, \forall x \in W, \forall Q_{2} c T_{x} W, \operatorname{dim} Q_{2}=2$, entã்: 


$$
\frac{n}{n-1} K_{1} \leqslant \frac{1}{\operatorname{vol}(W)} \int_{W} \gamma_{2} \leqslant \frac{n}{n-1} \quad K_{2}
$$

Alêm disso, se W ë uma variedade de Einstein temse :

$$
\frac{1}{\operatorname{vol}(W)} \int_{W} \gamma_{2}=\frac{1}{n-1} \rho
$$

onde $\rho$ é a curvatura de Ricci de W. Neste caso a integral da curvatura escalar das folhas não depende da fo 1heação".

Demonstração:

De acordo com o lema fundamental tem-se:

$$
\mathrm{d} \Psi_{1,0, \mathrm{n}}=\phi_{0,0, \mathrm{n}}-(-1)^{\mathrm{n}}(\mathrm{n}-1) \Psi_{2,0, \mathrm{n}} \wedge \theta_{\mathrm{n}+1}
$$

A expressão da curvatura escalar da folha será dacla uti lizando-se um referencial ortonormal adaptado da seguinte ma neira:

$$
\gamma_{2} \nu=-\frac{1}{n} ! \sigma \sum_{S_{n}} \operatorname{sgn}(\sigma) \bar{\Omega}_{\sigma(1) \sigma(2)} \wedge \theta_{\sigma(3)} \wedge \ldots \wedge \theta_{\sigma(n)} \wedge \theta_{n+1}
$$

onde $\bar{\Omega}_{i j}$ é a forma de curvatura da folha vista como subvarie dade regular de $w^{n+1}$ munida da mêtrica induzida pela inclusão da folha e $v$ representa a $(n+1)$ - forma volume de $w$.

Explicitamente: 


$$
\begin{aligned}
& \bar{\Omega}_{i j}=\omega_{i}^{*} n+1 \quad \Lambda \omega_{n+1}^{*}+\Omega_{i j}^{*}, \text { onde } \\
& \omega_{i n+1}^{*}=\lambda^{*}\left(\omega_{i n+1}\right), \quad \Omega_{i j}^{*}=\lambda^{*}\left(\Omega_{i j}\right),
\end{aligned}
$$

sendo que $\lambda: M_{x} \rightarrow W$ é a inclusão da folha que passa por $x$.

Teremos então:

$$
\begin{aligned}
& -\gamma_{2} \nu=\frac{1}{n !} \sum_{\sigma \in S_{n}} \operatorname{sgn}(\sigma)\left(\omega_{\sigma(1) n+1}^{*} \Lambda \omega_{n+1}^{*} \sigma(2)+\Omega_{\sigma(1) \sigma(2)}^{*}\right) \Lambda \\
& \Lambda \theta_{\sigma(3)} \Lambda \ldots \Lambda \theta_{\sigma(\mathrm{n})} \Lambda \theta_{\mathrm{n}+1}= \\
& =\frac{-1}{n !} \sum_{\sigma \varepsilon S_{n}} \operatorname{sgn}(\sigma) \quad \omega_{\sigma(1) n+1} \Lambda \omega_{\sigma(2) n+1} \Lambda \theta_{\sigma(3)} \Lambda \ldots \Lambda \theta_{\sigma(n)} \Lambda \theta_{n+1}+ \\
& +\frac{1}{n !} \sum_{\sigma \varepsilon S_{n}} \operatorname{sgn}(\sigma) \Omega_{\sigma(1) \sigma(2)} \Lambda \theta_{\sigma(3)} \Lambda \ldots \Lambda \theta_{\sigma(n)} \Lambda \theta_{n+1}= \\
& =-\frac{1}{n !} \quad \Psi_{2,0, n} \quad \Lambda \theta_{n+1}-\left[\frac{1}{\left(\begin{array}{l}
n \\
z
\end{array}\right)} \sum_{i<j=2}^{n} c_{i j}\right] v
\end{aligned}
$$

sendo que $c_{i j}$ è a curvatura seccional de W na direção do 2 - plano determinado por $\left\{e_{i}, e_{j}\right\}$.

O teorema de Stokes aplicado a (1) fornece:

$\int_{W} \Psi_{2,0, n} \quad \Lambda \theta_{n+1}=(-)^{n} \frac{1}{n-1} \int_{W} \phi_{0,0, n}$

Porêm, 


$$
\begin{aligned}
& (-1)^{\mathrm{n}} \phi_{0,0, \mathrm{n}}=(-1)^{\mathrm{n}} \sum_{\sigma \varepsilon \mathrm{S}_{\mathrm{n}}} \operatorname{sgn}(\sigma) \Omega_{\sigma(1) \mathrm{n}+1}{ }^{\Lambda \theta_{\sigma(2)}}{ }^{\Lambda . . \Lambda \theta_{\sigma(\mathrm{n})}} \\
& =\left[(n-1):{ }_{j=1}^{n} \quad c_{j} n+1\right] v
\end{aligned}
$$

Logo

$\int_{W} \Psi_{2,0, n} \wedge \theta_{n+1}=(n-2) ! \sum_{j=1}^{n} \quad c_{j} n+1$

Integrando-se os dois membros da igualdade (2) ob tem-se:

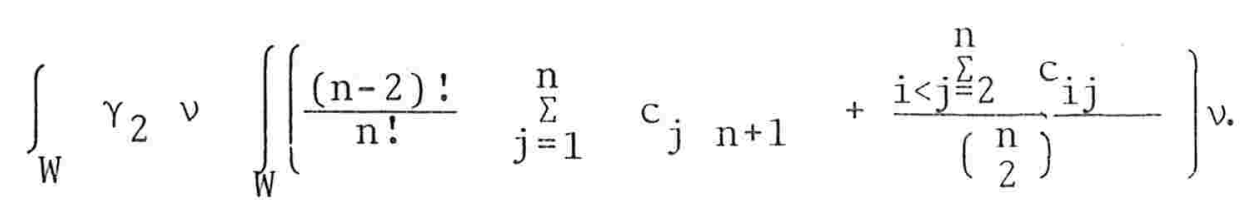

A desigualdade $K_{1} \leqslant c_{i j} \leqslant K_{2}$ implica:

$$
1 \leqslant i, j \leqslant n
$$




$$
\frac{n}{n-1} K_{1} \leqslant \frac{1}{\operatorname{vol}(W)} \int_{W} \gamma_{2} \nu \leqslant \frac{n}{n-1} K_{2}
$$

Se, alêm disso, w ë uma variedade de Einstein, a equação (3) nos dä:

$$
\begin{aligned}
& \frac{1}{\operatorname{vol}(W)} \int_{W} \gamma_{2}=\frac{1}{n-1} \rho \text {, posto que } \sum_{j=1}^{n} c_{j n+1}=\rho \text {, } \\
& \text { e } \sum_{i<j=2}^{n} c_{i j}=\frac{n-1}{2} \rho \quad \text { c.q.d. }
\end{aligned}
$$




\section{2 - Teorema \\ "Se existir um inteiro par $p, 2 \leq p \leq n$ tal que as cur} vaturas p-seccionais de $W$ sejam não nulas em todo ponto $x \in W$ e em todas as direções do espaço tangente então $W$ não admite folheações totalmente geodésicas de codimensão 1. Em particu lar, sôbre uma variedade riemanniama de curvatura seccional (2-secciona1) não nula não existem folheações totalmente geodés i cas de codimensão $1 "$.

\section{Demonstração:}

Suponhamos por absurdo que exista uma folheação totalmente geodēsica $F$ de codimensão 1 . Então, as formas ${ }^{*} \mathrm{k}, 2 \ell, \mathrm{n}$ são nulas. Assim seguindo a notação de 3.3., teremos:

$$
\begin{aligned}
& \Gamma_{p, n}=(-1)^{\frac{p}{2}+1} \frac{(p-1)(p-3) \ldots . .5 \cdot 3}{2.4 \cdot 6 \ldots \ldots(p-4)(p-2)} \phi_{0, p-2, n} \\
& \text { mas } \Gamma_{p, n} \vec{e} \text { exata. Logo. } \\
& \int_{W}(-1)^{\frac{p}{2}+1} \frac{(p-1)(p-3) \ldots .3}{2.4 .6 \ldots(p-4)(p-2)} \phi_{0, p-2, n}=0
\end{aligned}
$$

do geométrico:

Por outro 1 ado $\phi_{0, p-2, n}$ tem o seguinte significa

$$
\phi_{0, p-2, n}\left(e_{1}, e_{2}, \ldots, e_{n+1}\right)=(-1)^{\frac{p}{2}-1}(p-1):(n-p+1): \sum_{\substack{\Sigma \\ 1 \leq i_{1}<\ldots<i_{p-1}<n}} c_{i_{1} i_{2}} \cdots i_{p-1} n+1
$$

onde $c_{\dot{i}_{1}} i_{2} \ldots \dot{i}_{p-1}^{n+1}$ é a curvatura p-seccional na direção do plano determinado por $\left\{\mathrm{e}_{\mathrm{i}_{1}}, \mathrm{e}_{\mathrm{i}_{2}}, \ldots, \mathrm{e}_{\mathrm{i}_{\mathrm{p}-1}}, \mathrm{e}_{\mathrm{n}+1}\right\}$. Para uma defi 
nição das curvaturas p-seccionais ver [7].

Logo, (4) e (5) fornecem.

$$
\begin{aligned}
& \int_{W} i_{1 \leq i_{1}<i_{2}<\ldots<i_{p-1} \leq n} \quad c_{i_{1} i_{2} \ldots i_{p-1} n+1}=0 \\
& \text { Isto contradiz o fato } c_{i_{1} i_{2} \cdots i_{p-1} n+1} \neq 0 \text { em } W \text { por }
\end{aligned}
$$

que $W$ è conexo.

$$
c \cdot q \cdot d
$$

$$
\begin{aligned}
& 4.3-\text { Teorema: } \\
& \text { "Seja } \Xi_{2 k} ; 0 \leq 2 \mathrm{k} \leq \mathrm{n} a(n+1)-\text { forma sôbre W definida por } \\
& \Xi_{2 k}=-\Gamma_{2 k,+}(-1) \frac{(2 k-1)(2 k-3) \ldots .5 \cdot 3}{2 \cdot 4 \cdot 6 \ldots(2 k-2) \cdot 2 k}(n-2 k+1) \psi_{0,2 k, n}{ }^{\wedge \theta} n+1
\end{aligned}
$$

Então,

$\int_{W} \Xi_{2 k}=\alpha \int_{W} c_{2 k}$ onde $\alpha \vec{e}$ um coeficiente combinatório que só de pende de $k$ e de $n$; e $c_{2 k}$ designa a curvatura $2 k$-escalar de $W$. Em particular $\int_{W} \Xi_{2 k}$ só depende da métrica de W'

\section{Demonstração:}

${ }^{\Psi} 0,2 \mathrm{k}, \mathrm{n} \quad{ }^{\Lambda \theta_{\mathrm{n}+1}}=\sum_{\sigma \varepsilon \mathrm{Sn}} \operatorname{sgn}(\sigma) \Omega_{\sigma(1) \sigma(2)} \Lambda \ldots \ldots \Lambda$

$\Lambda \Omega_{\sigma(2 \mathrm{k}-1) \sigma 2 \mathrm{k})}{ }^{\Lambda \theta_{\sigma}} \sigma_{\sigma(2 \mathrm{k}+1)}{ }^{\Lambda \theta_{\sigma}}(2 \mathrm{k}+2){ }^{\Lambda} \ldots \mu_{\sigma}(\mathrm{n}){ }^{\Lambda \theta_{n+1}}$

Logo, $\vec{e}$ evidente que

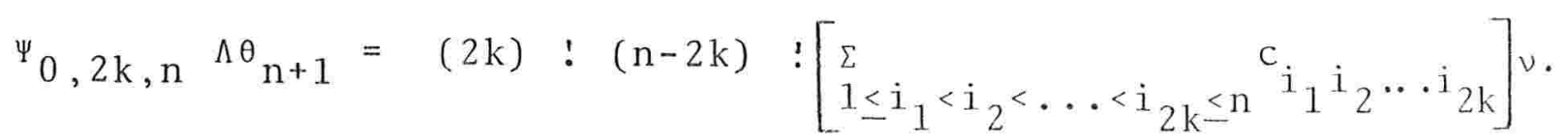

Aplicando-se a fórmula (5) do teorema 4.2 obtêm-se

o resultado desejado. $c \cdot q \cdot d \cdot$

\section{$\S 5 \cdot \underline{\text { Conclusão }}$}

Seja $H^{*}(W, \mathbb{R})$ a cohomologia de Rham de W C 
$\varepsilon(F) \in H^{\mathrm{n}}(W, \mathbb{R})$ a classe de Euler de F.Utilizando-se um referencial ortono mal adapatado local, $\varepsilon(F)$ se exprime da seguinte maneira (supon do-se que n é par):

$$
\begin{aligned}
& \varepsilon(F)=(-1)^{\frac{n}{2}} \frac{1}{n !} \operatorname{Pf}\left(\widetilde{\Omega}_{i j}\right) \text { onde } \\
& \widetilde{\Omega}_{i j}=\omega_{i} n+1{ }^{\Lambda \omega} n+1 j^{+} \Omega_{i j} \quad(\operatorname{ver}[7])
\end{aligned}
$$

\section{Logo}

$\varepsilon(F)=\frac{1}{n !}(-1)^{\frac{n}{2}} \sum_{\sigma \varepsilon S_{n}} \operatorname{sgn}(\sigma) \quad\left(\omega_{\sigma(1) n+1}{ }^{\Lambda \omega} n+1 \sigma(2){ }^{+\Omega} \sigma(1) \sigma(2)\right) \Lambda$

$\Lambda\left(\omega_{\sigma(3)} n+1 \omega_{n+1 \sigma(4)}{ }^{+\Omega} \sigma(3) \sigma(4)\right) \Lambda \ldots \ldots .$.

$\Lambda\left(\omega_{\sigma(n-1)} n+1 \omega_{n+1 \sigma(n)}+{ }^{\Lambda} \sigma(n-1) \sigma(n)\right.$

$$
\text { Logo, se } n=2 p, \text { temos }
$$

$\varepsilon(F)=\frac{1}{n !}\left(\Psi_{n, 0, n}-\left(\begin{array}{c}p \\ p-1\end{array}\right) \Psi_{n-2,2, n}+\left(\begin{array}{c}p \\ p-2\end{array}\right)_{\Psi_{n-4,4, n}}-\right.$

$-\ldots \ldots+(-1)^{p-1}\left(\begin{array}{c}p \\ 1\end{array}\right)_{\psi_{2}, n-2, n}+(-1)^{p_{\psi}} 0, n, n$

O teorema de Asimov (ver [1]), th.1) pode ser reenun ciado da seguinte maneira:

Se $w^{n+1}$ tem curvatura seccional constante, então $\varepsilon(F) \wedge \theta_{n+1} \varepsilon H^{n+1}(W, I R) n a ̃ o$ depende da folheação, mas somente de $n$ e da curvatura seccional constante igual a $c$.

o que fizemos no teorema 4.3 significa, para o ca so em que n é par, definir uma combinação linear standard ( no sentido que os coeficientes só dependem da dimensão n das fọ thas) $\bar{\Xi}_{n}$ das formas $\Psi_{2 r, n-2 r, n}$ de coeficientes racionais satis fazendo:

$\bar{E}_{n} \Lambda_{n+1}=\Xi_{\varepsilon} H^{n+1}(W, \mathbb{R})$ não depende da folheação $F$ mas sō 
mente da mëtrica riemanniana de W.

Entretanto as formas $\Xi_{n}$ não são fechadas em geral como o è a classe de Euler de $F, \varepsilon(F)$.

Uma propriedade interessante dos $\bar{\Xi}_{2 \mathrm{k}}$ e a seguin te:

$\int_{W} \Xi_{2 k} \vec{e} \circ 2 k$-êsimo coeficiente do polinômio em $t$ que forne ce o volume do tubo de $W$ se supusermos $W$ c $\mathbb{R}^{n+p}$.

Para um estudo do tubo de variedades riemannianas $\operatorname{ver}[6]$ 


\section{CAP ITULO II I}

\section{Folheações Ortogonais}

Analisaremos neste capitulo o comportamento das integrais de curvatura de folheações duas a duas ortogonais numa dada variedade riemanniana $W$.

\section{\$1. Folheações de Codimensão Um.}

Seja $W^{n+1}$ uma variedade riemanniana compacta co nexa e sem bordo de dimensão $n+1$ e sejam $F_{1}, F_{2}, \ldots, F_{n+1}$ fo 1heações sôbre $W$ duas a duas ortogonais e transversalmente o rientadas.

\section{1 - Teorema:}

"Sejam $\gamma_{2}{ }^{i}: W^{n+1} \rightarrow \mathbb{R}, i=1,2, \ldots, n+1$ dadas por: $\gamma_{2}{ }^{i}(x)=$ curvatura escalar da folha que passa por $x$ no ponto $x$ Então:

$$
\sum_{\substack{n+1 \\ i=1}}^{n+i}=-m \int_{W} k_{2} 2
$$

onde $\mathrm{K}_{2}$ é curvatura escalar de $\mathrm{W}$ e $\mathrm{m}$ è uma constante dada pe 
1 a dimensão de W.

Em particular, as integrais acima não dependem da escolha das folheações ortogonais $F_{i} "$ :

\section{Demonstração:}

Seja $\left\{e_{1}, e_{2}, \ldots, e_{n+1}\right\}$ um referencial global em $W$ que satisfaz:

$e_{i} I_{i} \quad i=1,2, \ldots, n+1$. Isto é possível porque as folheações $F_{i}$ são duas a duas ortogonais e transversalmente rientadas.

A fórmula (3) do teorema 4.1 do capítulo 2 nos dá:

$$
\int_{W} r_{2}^{i} \quad v=\int_{W}\left(\frac{(n-2)}{n !} \sum_{\substack{j \neq i \\
j=1}}^{n+1} c_{i j}+\frac{\sum_{i<j}+j \neq 1}{\left(\begin{array}{c}
n \\
2
\end{array}\right)} \quad c_{i j}\right.
$$

Assim,

$$
\sum_{i=1}^{n+1} \int_{W} \gamma \frac{i}{2} \nu=\int_{W}\left(\frac{(n-2) !}{n !} \cdot 2 \sum_{\substack{i<j=2 \\ \sum_{i j}}}^{c}+\frac{2}{n} \sum_{\substack{i<j \\ i=1}}^{n+1} c c_{i j}\right) \nu
$$

$\log 0$

$$
\begin{aligned}
& \sum_{i=1}^{n+1} \int_{W} \gamma_{2}^{i} \cdot \nu=2\left(\frac{(n-2)}{n !} !+\frac{1}{n}\right) \int_{W} K \\
& \text { pois } \sum_{\substack{i<j \\
i=1}}^{n+1} \quad c_{i j}=K \\
& c \cdot q \cdot d \text {. }
\end{aligned}
$$

\section{2 - Corolärio}

"Sejam $F_{1}, F_{2}, F_{3}$ três folheações transversalmente orientadas de codimensão 1 sôbre uma variedade $W^{3}$ de dimensão 3. Seja $k_{i}, i=1,2,3$, a curvatura de Gauss da folha que passa por $x$ no ponto $x$ correpondente à folheação $F$ : Então 


$$
\begin{aligned}
& \int\left(k_{1}+k_{2}+k_{3}\right) v \text { não depende da escolha das três folheações ortogo } \\
& \begin{array}{ll}
W^{3} \\
\text { nais." } & \text { c.c.d. }
\end{array}
\end{aligned}
$$

\section{§2. - Folheações Ortogonais de Dimensões Complemen tares.}

Seja $F$ uma folheação orientada e transversalmente orientada de codimensão p sôbre uma variedade riemanniana $W^{n+p}$ de dimensão $n+p$.

Um referencial ortonormal local adaptado a $F$ sera um referencial do tipo $\left\{e_{1}, e_{2}, \ldots e_{n}, e_{n+1}, \ldots, e_{n+p}\right\}$ onde:

$$
\begin{aligned}
& e_{1}, e_{2}, \ldots e_{n} \text { são tangentes às folhas de } F \text {, } \\
& , e_{n+1}, \ldots, e_{n+p} \text { são perpendiculares a } F .
\end{aligned}
$$

Sejam $\left\{\theta_{1}, \theta_{2}, \ldots, \theta_{n+p}\right\} ; \omega_{i j} ; \Omega_{i j} ; o$ correferencial, as formas de conexão e as formas de curvatura associadas a es te referencial.

$$
\begin{aligned}
& 2.1 \text { - Proposição } \\
& \text { "Seja no a }(n+p-1) \text { - forma local em } W \text { definida por: }
\end{aligned}
$$

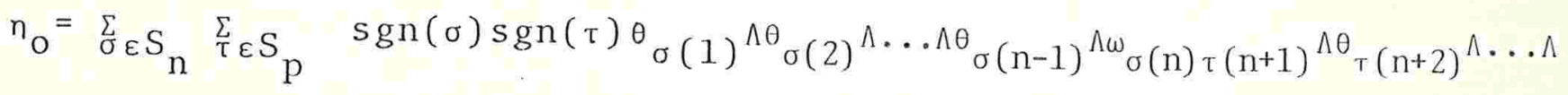

$$
\begin{aligned}
& \Lambda \theta_{\tau(n+p)}
\end{aligned}
$$

onde:

$S_{n} \vec{e}$ o grupo de permutações de $\{1,2, \ldots, n\}$

$s_{p}$ è o grupo de permutações de $\{n+1, n+2, \ldots, n+p\}$.

Neste caso, no é uma forma global em $W^{\prime \prime}$

Demonstração

Seja $\left\{\overline{\mathrm{e}}_{1}, \overline{\mathrm{e}}_{2}, \ldots, \overline{\mathrm{e}}_{n}, \overline{\mathrm{e}}_{n+1}, \overline{\mathrm{e}}_{n+2}, \ldots, \overline{\mathrm{e}}_{n+p}\right\}$ um outro 
referencial adaptado coerente com a orientação de $F$ e com a orientação transversal de F.

\section{Teremos:}

$$
\begin{aligned}
& \bar{e}_{i}=\sum_{j=1}^{n} a_{i j} e_{j}, 1 \leq i, j \leq n \\
& \bar{e}_{\alpha}=\sum_{\beta=n+1}^{n+p} b_{\alpha \beta} e_{\beta}, n+1 \leq \alpha, \beta \leq n+p \\
& \bar{\theta}_{i}=\sum_{j=1}^{n} a_{i j}{ }^{\theta}, \quad 1 \leq i, j \leq n \\
& \bar{\theta}_{\alpha}=\begin{array}{c}
n+p \\
= \\
\beta=n+1
\end{array} \quad b_{\alpha \beta} \theta_{\beta}, n+1 \leq \alpha, \beta \leq n+p
\end{aligned}
$$

onde $\left(a_{i j}\right)$ e $\left(b_{\alpha \beta}\right)$ são matrizes ortogonais e $\operatorname{det}\left(a_{i j}\right)=1=\operatorname{det}\left(b_{\alpha \beta}\right)$

$$
\text { Teremos também: }
$$

$$
\begin{aligned}
& \bar{\omega}_{i \alpha}(u)=\left\langle\nabla_{u} \bar{e}_{i}, \bar{e}_{\alpha}\right\rangle=-\sum_{\beta=n+1}^{n+p} b_{\alpha \beta}\left\langle\bar{e}_{i}, \nabla_{u} e_{\beta}\right\rangle= \\
& =\sum_{\beta=n+1}^{n+p} \sum_{j=1}^{n} b_{\alpha \beta} a_{i j}\left\langle\nabla_{u} e_{j}, e_{\beta}\right\rangle= \\
& =\sum_{\beta=n+1}^{n+p} \sum_{j=1}^{n} a_{i j} b_{\alpha \beta} \omega_{j \beta}(u)
\end{aligned}
$$

\section{$\log 0$}

$$
\begin{aligned}
& \bar{n}_{0}=\sum_{\sigma \varepsilon S_{n}} \sum_{\tau \varepsilon S_{p}} \operatorname{sgn}(\sigma) \operatorname{sgn}(\tau) \bar{\theta}_{\sigma(1)}{ }^{\Lambda \bar{\theta}} \sigma(2) \Lambda \ldots \Lambda \bar{\theta}_{\sigma(n-1)} \Lambda \\
& \Lambda \bar{\omega}_{\sigma(\mathrm{n}) \tau(n+1)}{ }^{\Lambda \bar{\theta}_{\tau}}{ }_{\tau(n+2)} \Lambda \ldots \Lambda \bar{\theta}_{\tau} \quad(n)= \\
& \left.\left.\equiv \sum_{\sigma \in S_{n}} \sum_{\tau \in S_{p}} \operatorname{sgn}(\sigma) \operatorname{sgn}(\tau) \underset{j=1}{\left(\sum_{j=1}^{n}\right.} a_{\sigma(1) j}{ }_{j}\right) \stackrel{n}{n} \sum_{j=1} a_{\sigma(2) j} \theta_{j}\right) \Lambda
\end{aligned}
$$


$\left.\Lambda \ldots \Lambda \sum_{j=1}^{n} a_{\sigma(n-1) j} \theta_{j}\right) \Lambda \sum_{j=1}^{n} \sum_{\beta=n+1}^{n+p} a_{\sigma(n) j} b_{\tau(n+1) \beta}{ }^{\omega}{ }_{j \beta} \Lambda$

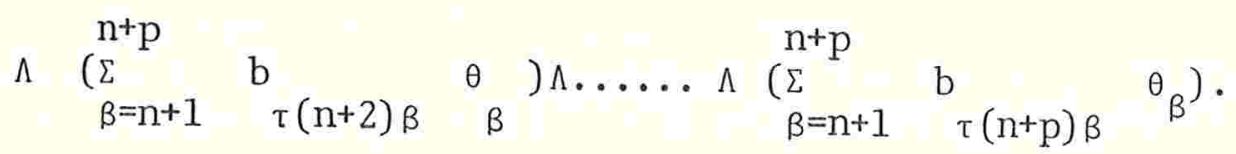

Fixando-se primeiro $\tau$ e depois o e utilizando- se

os mesmos argumentos da proposição 3.1 do capĩtulo 2 obtemos:

$$
\bar{n}_{0}=n_{0} \quad \text { c.q.d. }
$$

\section{2 - Definição}

Sejam $\eta_{2,0}, n_{0,2}$ e $\mu_{0}(n+p)$-formas 1ocais em $W$ de finidas por:

$\eta_{2,0}=\sum_{\sigma \varepsilon S_{n}} \sum_{\tau \varepsilon S} \operatorname{sgn}(\sigma) \operatorname{sgn}(\tau) \sum_{k=n+1}^{n+p} \omega_{\sigma(1) k} k^{\Lambda \omega} \omega_{(2)} \Lambda \theta_{\sigma(3)} \wedge \ldots \theta_{\sigma(n-1)}$

${ }^{\Lambda \theta_{\sigma(n)}}{ }^{\Lambda \theta} \tau(n+1){ }^{\Lambda \ldots \Lambda \theta_{\tau(n+p)}}$

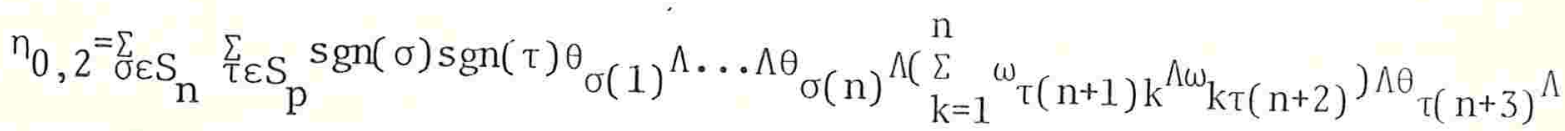

$\Lambda \theta_{\tau(n+4)} \Lambda \ldots \Lambda \theta_{\tau(n+p)}$

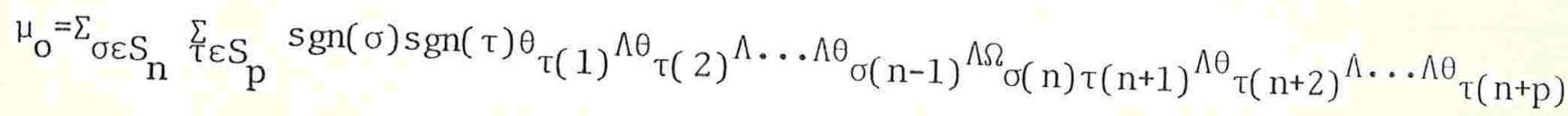

\section{3 - Proposição}

"n $2,0, \quad n_{0,2}$ e $\mu_{0}$ são globais "

Demonstração:

Anảloga à proposição 2.1

c.q.d. 


\section{$2.4-\underline{\text { Lema }}$}

$$
\text { "d n } n_{0}=(-1)^{n}(n-1):(p-1):\left(n_{2,0}+n_{0,2}\right)+(-1)^{n+1} \mu_{0} "
$$

Demonstração:

$$
\mathrm{dn} n_{0}=\mathrm{X}+\mathrm{Y}+\mathrm{Z} \text { onde: }
$$

$$
\begin{aligned}
& X=(n-1) ! \sum_{\sigma \varepsilon S_{n}} \sum_{\tau \varepsilon S} \operatorname{sgn}(\sigma) \operatorname{sgn}(\tau) \operatorname{d\theta } \theta_{\sigma(1)}{ }^{\Lambda \theta_{\sigma}}(2) \Lambda \ldots \Lambda \theta_{\sigma(n-1)} \Lambda \\
& \Lambda \omega_{\sigma(n) \tau(n+1)}{ }^{\Lambda \theta_{\tau}}{ }_{\tau(n+2)} \Lambda \ldots \Lambda \theta_{\tau(n+p)} \\
& Y=(-1)^{n-1} \sum_{\sigma \varepsilon S_{n}} \sum_{\tau \in S} S_{p} \operatorname{sgn}(\sigma) \operatorname{sgn}(\tau) \theta_{\sigma(1)}{ }^{\Lambda \theta} \sigma(2) \Lambda \ldots \Lambda \theta_{\sigma(n-1)} \Lambda
\end{aligned}
$$$$
\Lambda \mathrm{d} \omega_{\sigma(n) \tau(n+1)}{ }^{\Lambda \theta_{\tau}}{ }_{\tau(n+2)}{ } \ldots \Lambda \theta_{\tau(n+p)}
$$$$
Z=(-1)^{n}(p-1) ! \sum_{\sigma \varepsilon S_{n}} \sum_{\tau \varepsilon S_{p}} \operatorname{sgn}(\sigma) \operatorname{sgn}(\tau) \theta_{\sigma(1)} \wedge \ldots \Lambda \theta_{\sigma(n-1)} \Lambda
$$$$
\Lambda \omega_{\sigma(n) \tau(n+1)} \Lambda d \theta_{\tau(n+2)}{ }^{\Lambda \theta_{\tau}(n+3)} \Lambda \ldots \Lambda \theta_{\tau(n+p)} \cdot
$$

Desenvolvendo $\mathrm{X}$ obtemos:

$$
\mathrm{X}=\mathrm{X}_{1}+\mathrm{X}_{2} \quad \text { onde : }
$$

$x_{1}=(-1)^{n}(n-1): \sum_{\sigma \varepsilon} S_{n} \sum_{\tau \varepsilon} S_{p} \operatorname{sgn}(\sigma) \operatorname{sgn}(\tau) \omega_{\sigma(1) \sigma(n)}{ }^{\wedge} \theta_{\sigma(2)} \Lambda \ldots \Lambda \theta_{\sigma(n-1)} \Lambda$

$$
\begin{aligned}
& { }_{\sigma}^{\Lambda \theta_{\sigma}(n)}{ }^{\Lambda \omega_{\sigma(}(n) \tau(n+1)}{ }_{\tau(n+2)}^{\Lambda \theta}{ } \ldots \Lambda_{\tau} \theta_{\tau(n+p)} \\
& X_{2}=(-1)^{n}(n-1):(p-1): n_{2,0} .
\end{aligned}
$$

Por outro lado é claro que $Y=(-1)^{n+1} \mu_{0}+Y_{1}, \quad$ onde:

$Y_{1}=(-1)^{n+1} \sum_{\sigma \varepsilon S_{n}} \sum_{\tau \varepsilon S_{p}} \operatorname{sgn}(\sigma) \operatorname{sgn}(\tau) \theta_{\sigma(1)} \Lambda \ldots \theta_{\sigma(n-1)} \Lambda$

$\Lambda\left(\sum_{k=1}^{n+p} \omega_{\sigma(n) k}{ }^{\Lambda \omega_{k}} k(n+1)\right) \quad \Lambda \theta_{\tau(n+2)} \Lambda \Delta_{\tau}(n+p)$

$$
\text { Por sua vez, } Y_{1}=Y_{11}+Y_{12} \text { onde }
$$

$\dot{Y}_{11}=-X_{1} e$ 


$$
\begin{aligned}
& \mathrm{Y}_{12}=(-1)^{\mathrm{n}+1}(\mathrm{p}-1): \sum_{\sigma \varepsilon \mathrm{S}_{\mathrm{n}}} \sum_{\tau \in S_{p}} \operatorname{sgn}(\sigma) \operatorname{sgn}(\tau) \theta_{\sigma(1)} \Lambda \ldots \Lambda \theta_{\sigma(\mathrm{n}-1)} \Lambda \\
& \Lambda \omega_{\sigma(n) \tau(n+1)}{ }^{\Lambda \omega_{\tau}} \tau(n+2) \tau(n+1){ }_{\tau(n+2)}^{\Lambda \theta_{\tau}} \tau(n+3){ }^{\Lambda} \ldots \Lambda \theta_{\tau}(n+p) \\
& \text { Para Z temos: } \\
& Z=(-1)^{\mathrm{n}}(\mathrm{p}-1): \sum_{\sigma \varepsilon S_{n}} \sum_{\tau \varepsilon S} \operatorname{sgn}(\sigma) \operatorname{sgn}(\tau) \theta_{\sigma(1)} \Lambda \ldots \Lambda \theta_{\sigma(n-1)} \Lambda \\
& \left.\Lambda \omega_{\sigma(n) \tau(n+1)} \Lambda \sum_{k=1}^{n+p} \omega_{\tau(n+2) k} \Lambda \theta_{k}\right) \Lambda \theta_{\tau(n+3)} \Lambda \ldots \Lambda \theta_{\tau(n+p)} \\
& \text { Logo, } \mathrm{Z}=\mathrm{Z}_{1}+\mathrm{Z}_{2} \text { onde: } \\
& \mathrm{z}_{1}=(-1)^{\mathrm{n}}(\mathrm{p}-1):(\mathrm{n}-1): \mathrm{n}_{0,2} \quad \mathrm{e} \\
& Z_{2}=-Y_{12}
\end{aligned}
$$

Assim,$$
d n_{0}=X+Y+Z=(-1)^{n}(n-1):(p-1): n_{2,0}+
$$$$
+(-1)^{n}(p-1):(n-1): n_{0,2}+(-1)^{n+1} \mu_{0} \quad c \cdot q \cdot d \cdot
$$

Vejamos agora uma primeira generalização do teore ma de Asimov para o caso de codimensão $>1$.

$$
\begin{aligned}
& 2.5-\underline{\text { Teorema }} \\
& \text { "Seja } \mathrm{T}^{\mathrm{n}}=\underbrace{\text { 'x }^{\prime} \mathrm{x} \ldots \mathrm{xS}}_{\mathrm{S} \text { vezes }} ; \text {; o toro "flat" e sejam } F_{1} \text {, }
\end{aligned}
$$

$F_{2}$ duas folheações ortogonais em $T^{n}$ tais que dim $F_{1}+$ dim $F_{2}=n$.

Suponhamos que $F_{1}$ e $F_{2}$ são transversalmente orien tadas.

$$
\text { Então } \int_{T^{n}} \gamma_{2}{ }^{1} v=-\frac{\left(\begin{array}{l}
n \\
2
\end{array}\right)}{\left(\begin{array}{l}
p \\
2
\end{array}\right)} \int_{T^{n}} \gamma_{2}^{2} v
$$


onde $r_{2}{ }^{1}$ e $r_{2}{ }^{2}$ são as curvaturas escalares das folhas de $F_{1}$ e $F_{2}$ respectivamente."

\section{Demonstração:}

As curvaturas seccionais de $\mathrm{T}^{\mathrm{n}}$ são identicamente nulas, $\operatorname{logo} \Omega_{i j}=0 \quad \forall i, j \quad 1 \leq i, j \leq n+p$. Assim, $\mu_{0}=0$

Então, pe1o 1ema 2.4,

$$
n_{2,0}+n_{0,2} \quad \text { è exata }
$$

Por outro 1ado, sabemos que:

$$
\begin{aligned}
& n_{2,0}=-2(n-2): p: r_{2}{ }^{1} \nu \text { e } \\
& n_{0,2}=-2 n:(p-2): r_{2}^{2} \nu
\end{aligned}
$$

onde $v \vec{e}$ o elemento de volume de $W$.

Pelo teorema de Stokes temos:

$$
\int_{W} 2(n-2) ! p ! \gamma_{2}^{1} \quad \nu=-\int_{W} 2 n !(p-2): \gamma_{2}^{2} \nu,
$$

, ou equivalentemente

$$
\int_{W} \gamma_{2}^{1} v=-\frac{\left(\begin{array}{l}
n \\
2
\end{array}\right)}{p} \int_{W} \gamma_{2}{ }^{2} v \quad c \cdot q \cdot c^{\prime} \cdot
$$

Mais geralmente temos:

\section{6 - Proposição}

"Seja $\mathrm{W}^{\mathrm{n}+1}$ uma variedade riemanniana compacta co nexa e sem bordo e $F_{1}, F_{2}$ duas folheações ortogonais trans versalmente orientadas e tais que 
$\operatorname{dim} F_{1}+\operatorname{dim} F_{2}=n$. Então:

$$
\begin{aligned}
& 2(\mathrm{n}-2): \mathrm{p}: \int_{W} \sigma_{2}{ }^{1}+2 n:(\mathrm{p}-2): \int_{W} \sigma_{2}{ }^{2}= \\
& =\int_{W} \sum_{\substack{1 \leq i \leq p \\
n+1 \leq \alpha \leq n+p}} c_{i \alpha} \quad \text { c. r. } \cdot d .
\end{aligned}
$$

onde :

$c_{i \alpha}$ é a curvatura seccional de W na direção do plano determina do pelos vetores $e_{i}, e_{\alpha}$ pertencentes ao referencial ortonormal adaptado $\left\{e_{1}, e_{2}, \ldots, e_{n}, e_{n+1}, e_{n+2}, \ldots, e_{n+p}\right\}$ coerente com as orien tações.

, $\sigma_{2}^{1}, \sigma_{2}^{2}$ são as curvaturas escalares extrínsecas(dadas pela $2^{\text {a }}$ forma fundamental) das folhas de $F_{1}$ e $F_{2}$ respectivamente".

Demonstração:

Temos:

$$
\begin{aligned}
& \eta_{2,0}=-2(n-2): p: \sigma_{2}^{1} v \\
& n_{0,2}=-2 n:(p-2): \sigma_{2}^{2} v \\
& \left.\mu_{0}=-(n-1):(p-1): \sum_{\substack{1 \leq i \leq n \\
n+1 \leq \alpha \leq n+p}} \quad c_{i \alpha}\right) v
\end{aligned}
$$

O 1ema 2.4 e o teorema de Stokes nos dão:

$$
\int \mu_{0}=(n-1):(p-1): \int\left(n_{2,0}+n_{0,2}\right)
$$

W

$$
\text { W }
$$

$$
\text { Logo , }
$$

$$
\int_{W}^{\left.\left.\sum_{\substack{1 \leq 1 \leq n \\ n+1 \leq \alpha \leq n+p}} c_{i \alpha}\right) v=\int_{W} 2(n-2): p: \sigma_{2}+2 n:(p-2): \sigma_{2}^{2}\right) v} c
$$




\section{$2.7-$ Teorema}

"Nas mesmas condições dos Teoremas precedentes, se $w^{n+p}$ possui curvatura seccional constante então:

$$
2(n-2): p: \int_{W} \gamma_{2}^{1} \nu+2 n:(p-2): \int_{W} \gamma_{2}^{2} v=
$$

$=\operatorname{npc} \operatorname{vol}(W)$

Em particular,

$$
\int_{W} \gamma_{2}{ }^{1} \nu+\frac{\left(\begin{array}{c}
\mathrm{n} \\
2
\end{array}\right)}{\left(\begin{array}{l}
\mathrm{p} \\
2
\end{array}\right)} \int_{W} \gamma_{2}{ }^{2} \nu \text { não depende da escolha das duas }
$$

folheações ortogonais $F_{1}$ e $F_{2}$ "'

\section{Demonstração}

Basta observar que no caso de curvatura constante vale:

$$
\begin{aligned}
& c_{i \alpha}=c \quad 1 \leq i \leq n \quad n+1 \leq \alpha \leq n+p \quad e \\
& \gamma_{2}^{1}=\sigma_{2}^{1}+c, \\
& \gamma_{2}{ }^{2}=\sigma_{2}{ }^{2}+c
\end{aligned}
$$

e utilizar a proposição 2.6. c.q.d.

O teorema que enunciaremos a seguir fornece uma obstrução topológica à existência de folheações ortogonais de dimensões complementares em espaços de curvatura positiva quando uma das folheações ê tal que as folhas são subvarieda des mínimas do espaço ambiente. 
Ta1 resultado ap1ica-se, por exemplo, ao caso das esferas de curvatura constante. Um estudo detalhado de folhe ções satisfazendo condições geomëtricas particulares pode ser visto em [13].

\section{$2.8-\underline{\text { Teorema }}$}

"Seja $\mathrm{W}^{\mathrm{n}+2}$ uma variedade riemanniana de dimensão n+2 compacta, conexa, e sem bordo, de curvatura seccional es tritamente positiva. Sejam $F_{1}, F_{2}$ duas folheações ortogonais transversalmente orientadas tais que $\operatorname{dim} F_{1}=\mathrm{n}, \operatorname{dim} F_{2}=2$. Suponhamos que $F_{1}$ é tal que as folhas são subvariedades mín mas de W. Então,

$$
\begin{aligned}
& \varepsilon\left(F_{2}\right) \neq 0 \quad, \text { onde: } \\
& \varepsilon\left(F_{2}\right) \in H^{2}(W, \mathbb{R}) \text { è a classe de Euler da distribuição } \\
& \text { tangente a } F_{2} \text { ". }
\end{aligned}
$$

Demonstração:

Seja $\left\{e_{1}, e_{2}, \ldots e_{n}, e_{n+1}, e_{n+2}\right\}$ um referencial or togonal adaptado.

$$
\text { Seja } \nu_{1}=\theta_{1} \Lambda \theta_{2} \Lambda \ldots \theta_{n} \text { a forma caracteristica }
$$

de $F_{1}$.

$$
\begin{aligned}
& \text { Como } \left.F_{1} I=F_{2} \text { è integrável temos: (ver }[4],[8],[11]\right) \\
& \mathrm{d} \nu_{1}=0, \log 0\left[\nu_{1}\right] \text { \& } \mathrm{H}^{\mathrm{n}}(W, \mathbb{R}) .
\end{aligned}
$$

A classe de Euler de $F_{2}$ é representada na cohomo 
logia de Rham pela seguinte 2 - forma : (ver [7])

$$
\varepsilon\left(F_{2}\right)=\Omega_{n+1} n+2+\sum_{k=1}^{n} \omega_{n+1} \quad \Lambda \omega \quad k n+2
$$

(Note-se que a orientação de $F_{2}$ está fixada).

$$
\text { Assim, seja }
$$

$\left[\begin{array}{lll}\varepsilon\left(F_{2}\right) \Lambda & \nu_{1}\end{array}\right] \varepsilon H^{n+2}(W, \mathbb{R})$ o "cup product" dessas duas for mas. Pelo teorema de Rham temos:

$$
\begin{aligned}
& {\left[\begin{array}{lll}
\varepsilon\left(F_{2}\right) & \Lambda & \nu_{1}
\end{array}\right] \neq 0 \quad \rightleftarrows \quad \int_{W} \varepsilon\left(F_{2}\right) \wedge \nu_{1} \neq 0} \\
& \text { Por outro lado temos: } \\
& \varepsilon\left(F_{2}\right) \quad \Lambda v_{1}=\Omega_{\mathrm{n}+1} \mathrm{n}+2 \quad \Lambda \theta_{1} \Lambda \theta_{2} \Lambda \ldots \Lambda \theta_{\mathrm{n}}+ \\
& +\sum_{\mathrm{k}=1}^{\mathrm{n}} \quad \omega_{\mathrm{n}+1} \mathrm{k} \quad{ }^{\Lambda \omega_{\mathrm{k}} \mathrm{n}+2} \quad \Lambda \theta_{1} \Lambda \theta_{2} \Lambda \ldots \Lambda \theta_{\mathrm{n}}
\end{aligned}
$$

Mas ,

$$
\begin{aligned}
& \Omega_{n+1} \quad n+2 \quad{ }^{\Lambda \theta_{1}}{ }_{1} \theta_{2} \Lambda \ldots \Lambda \theta_{n}= \\
& =-c_{n+1} n+2{ }^{\theta}{ }_{1} \Lambda \theta_{2} \Lambda \ldots \Lambda \theta_{n} \Lambda \theta_{n+1}{ }_{n}{ }_{n+2}= \\
& =-c_{n+1} n+2 v \quad \text { onde: }
\end{aligned}
$$

$c_{n+1} n+2$ e a curvatura seccional de w na dire ção do plano gerado por $e_{n+1}$ e $e_{n+2}$, 
, v é o elemento de volume de W.

Tambẻm,

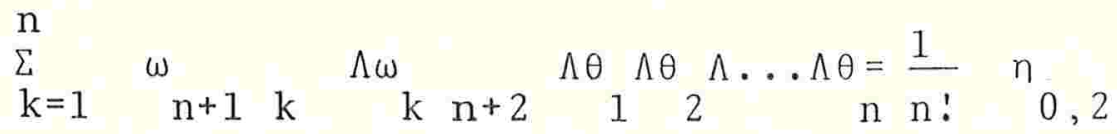

Assim,

$\int_{W} \varepsilon\left(F_{2}\right) \Lambda \nu_{1}=-\int_{W} c_{n+1} n+2 \nu+\frac{1}{n}: \int_{W} n_{0,2}$

Aplicando-se o lema 2.4 e o teorema de Stokes, te

mos:

$\int_{W} \varepsilon\left(F_{2}\right) \Lambda \nu_{1}=-\int_{W} c_{n+1} n+2 \nu \frac{1}{n !} \int_{W} \mu_{0}-\frac{1}{(n-1) !(p-1) !} \int_{W} n$

Porëm, sabemos que

$$
\mu_{0}=-(n-1):(p-1) !\left(\begin{array}{ll} 
& 1 \leq i \leq n \\
& n+1 \leq \alpha \leq n+p
\end{array} \quad c_{i \alpha}\right) v
$$

Como $\mathrm{c}_{i \alpha}>0$ temos

$\int \mu_{0}<0$

W

Tambêm, como $F_{1} \bar{e}$ tal que as folhas são subvarie dades minimas de $W$, temos:

$$
\sigma_{2}{ }^{1} \leq 0 \text { Logo, }
$$




$$
\int_{W} n_{2,0}=-\int_{W} 2(n-2): p: \sigma_{2}{ }^{1} \nu \geq 0
$$

Como $\mathrm{c}_{\mathrm{n}+1} \quad \mathrm{n}+2>0$ temos

$\int_{W} \varepsilon(F) \Lambda \nu_{1}<0$

logo $\varepsilon\left(F_{2}\right) \neq 0 \quad$ em $H^{2}(W, \mathbb{R}) \quad$ c.c.d.

\section{9 - Coro1ärio}

"Seja F uma folheação transversalmente orientada de codimensão 2 sôbre a esfera $S^{n} C \mathbb{R}^{n+1}$.

Se $F$ é tal que as folhas são subvariedades mini mas de $S^{n}$ então sua distribuição normal $F^{1} \operatorname{cTS}^{n}$ não é integrá ve1."

Demonstração:

Basta notar que

$0=\varepsilon(F) \varepsilon H^{2}\left(S^{n}, \quad \mathbb{R}\right)=0$

e ap1icar o teorema $2.8 \quad$ c.c.d. 


\section{B I BL IOGRAF IA}

(1) D. Asimov: "Average Gaussian curvature of leaves of a foliation" B.A.M.S. - Jan. 1978 .

(2) F.Brito, R. Langevin e H. Rosenberg: "Integrales de courbure sur des varietés feuilletées". C.R.A.S., T. 285(oct.1977).

(3) S.S.Chern: "A simple intrinsic proof of the Gauss-Bonnet formula for closed riemannian manifolds".

Annals of Math, vol.45, n־4 (1944).

(4) A.Haefliger: "Some remarks on foliations with minimal 1eaves" Journal of Diff.Geom. vol.15 n² (jun.1980).

(5) R. Langevin: "Feuilletages tendus". Bul1.Soc.Math.Fr. $n^{\circ} 107$ (1979) .

(6) R. Langevin: Thèse d'ëtat

Publications mathematiques d'Orsay. n 80-03 (jun.1980).

(7) J.W.Milnor, J.Stasheff: "Lectures on characteristic classes" Princeton University Press-1974, (Annals of Math.Studies).

(8) H. Rummler: Quelques notions simples en gêomêtrie riemanni enne et leurs applications aux feuilletages compacts. Comm. Math. He1v. vol.54, Fasc.2 (1979).

(9) L. Santaló: "Integral geometry and geometric probability" Encyclopedia of Mathematics and Its Applications. Add. Wesley Pub1. Co. (1976).

(10) M.Spivak: "Differential Geometry" Publish or Perish, Inc. (1975). 
(11) D. Sullivan: "A homological characterization of foliations consisting of minimal leaves". Comm.Math. Helv. 54 (1979).

(12) J. Thorpe: "Sectional curvatures and characteristic classes" Ann. of Math. vol.80 (1964).

(13) P. Walczak: "On foliations with leaves satisfying some geometrical conditions". Inst.of Math.Polish.Acad. of Sciences. Prep. n' 210 (1980).

(14) J.A.Wo1f: "Spaces of constant curvature" Mac Graw-Hil1 Book Company (1976). 